\title{
Spatial quantum correlations in the fluorescence of traveling-wave second-harmonic generation
}

\author{
Pierre Scotto \\ Instituto Mediterráneo de Estudios Avanzados, IMEDEA (CSIC-UIB), Campus Universitat Illes Balears, \\ E-07071 Palma de Mallorca, Spain
}

(Received 14 December 2002; published 29 September 2003)

\begin{abstract}
We investigate theoretically the spatial quantum correlations of the light produced by spontaneous emission in type-I second-harmonic generation in the traveling-wave configuration. It is first shown that spontaneous emission occurs at both fundamental and second-harmonic frequency. Considering the quantum fluctuations of the intensities collected in symmetrical parts of the far-field plane, nonclassical correlations below the shot noise are predicted not only at fundamental frequency, but also at second-harmonic frequency. The latter cannot be traced back to any twin-photon emission mechanism, but are generated by a secondary process acting on twin photons. This mechanism also creates correlations between fundamental and second-harmonic field, at a given transverse wave number, and at opposite wave numbers. The analysis of a simplified few-mode model, on a quantum level, provides a good qualitative understanding of these correlations.
\end{abstract}

DOI: 10.1103/PhysRevA.68.033814

PACS number(s): 42.50.Lc, 42.50.Dv, 42.65.Ky

\section{INTRODUCTION}

The concept of twin photons is of a disarming simplicity: when, in a nonlinear (NL) optical system, an elementary process leads to the creation of pairs of distinguishable photons, these are referred to as "twin photons." The simplest example is parametric down-conversion, in which photons of an intense pump beam propagating through a $\chi^{(2)}$-NL crystal can disintegrate, under conservation of energy and momentum, into pairs of photons of lower energy [1-3]. Quantum mechanically, these highly correlated twin photons are described by an entangled state [4-6], and therefore are connected with the most fundamental issues of quantum mechanics [7-9]. At the same time, they turn out to have numerous technological applications in two-photon imaging [10], quantum lithography [11], quantum cryptography $[12,13]$, quantum teleportation $[14,15]$, and quantum computation [16].

By means of an optical resonator, one can increase the efficiency of a twin-photon generation process [17], and eventually obtain macroscopic numbers of photon pairs, generally referred to as "twin beams." As a consequence of the underlying photon pair generation mechanism, the level of quantum noise in the difference of the intensities of twin beams drops below the standard quantum limit, as predicted for the nondegenerate optical parametric oscillator (OPO) [17-19] and demonstrated experimentally [20]. Spatially separated twin beams, translating into two symmetrical light spots in the far field [21], have been predicted for the degenerate $\mathrm{OPO}$ [22], in which a pump photon may decay into two half-frequency photons propagating in opposite directions in the transverse plane, and for a vectorial Kerr model in the self-defocusing case [23-26], where two linearly polarized pump photons annihilate under creation of two counterpropagating photons with the orthogonal polarization. Close to the pump threshold, at which twin-beams build up in the optical cavity, twin photon generation is the dominant process and the correlations of the quantum fluctuations of the twin beam intensities are directly related to energy and momentum conservation. Moreover, a three-mode model [27,22], describing the coupled dynamics of the two modes associated with the twin beams and the pump mode, provides a good understanding of the quantum properties of the fields [25,26].

However, neither this simplified description of the dynamics of coupled quantum fields, nor the resulting intuitive explanation of the predicted correlations is possible in numerous situations occurring in NL optical systems, namely, when the nonlinear field dynamics couples more than three field modes. The properties of quantum correlations in the case of "multi-mode interaction," which have recently attracted an increased theoretical interest, are addressed in the present paper. More specifically, we focus here on the quantum properties of traveling-wave second-harmonic generation (TWSHG), which is probably the simplest system, in which the consequences of "multimode interaction" can be studied at a quantum level. As a matter of fact, the nonlinear field dynamics leads to a coupling of four radiation modes: two of them can be identified with a pair of twin photons, but now each of these modes turns out to be coupled to another radiation mode through a secondary process, which is in the case of TW-SHG a frequency up-conversion process. The consequences of this secondary process on the spatial quantum properties of the system will be the main focus of this paper.

Previous investigations of twin-beam correlations in the presence of "multimode interaction" have been carried out for various NL optical systems and revealed a rich phenomenology. In the OPO above threshold, the twin-beam character was found to be largely preserved, not only for pump values slightly above threshold [28], despite the increasing number of field modes displaying a macroscopic intensity [21,29], but also far above threshold, where spatially disordered structures emerge [30]. On the contrary, noise sustained structures, characteristic of a convective instability $[31,32]$, were shown to destroy any kind of twin-beam correlations [33].

A rich multimode dynamics may emerge as a consequence of a transverse variation of the pump of an OPO [34] or, as in the scalar Kerr model, of an instability leading to a hexagonal pattern [35]. In the latter case, conservation laws 
imply a perfect noise reduction of combinations of the intensities of groups of four among the six off-axis far-field spots $[36,37]$. The fluctuations of the intensities of individual spots, however, were shown to display the strongest correlations not at opposite wave vectors, as suggested by a naive picture based on twin-photon emission, but for those forming a $120^{\circ}$ angle [38]. In the case of a square pattern, modes associated with critical wave vectors forming an angle of $\pi / 2$ were even found to be anticorrelated [39]. Similarly, a multimode description is necessary in the case of intracavity SHG, since at a given threshold, both fundamental and second-harmonic field may destabilize at a finite wave number, while below this threshold, only the homogeneous mode at each frequency displays a macroscopical intensity. Nonclassical correlations of the intensity fluctuations at symmetrical far-field points have been predicted not only at fundamental frequency, but also at second-harmonic frequency [40]. The origin of the latter correlations, which cannot be explained in terms of twin-photon emission, since no elementary process can generate two counterpropagating second-harmonic waves, has not been so far completely elucidated.

In this paper, we consider SHG in the traveling-wave configuration and investigate the spatial quantum correlations displayed by the fields generated by spontaneous emission. As we will show, spontaneous emission in TW-SHG generates field waves with nonvanishing transverse wave vectors, both at fundamental and second-harmonic ( $\mathrm{SH}$ ) frequency. These transverse modes being originally in the vacuum state, field generation occurs "spontaneously," as the result of the interaction of the vacuum fluctuations of these modes with the strong homogeneous waves at fundamental frequency (pumping) and SH frequency (frequency up-conversion of the pump through SHG). The study of the spatial quantum properties of TW-SHG presents a twofold interest: from a practical point of view, nonlinear optical systems in the single-pass configuration are interesting because their temporal and spatial bandwidths are generally much larger than the ones of their cavity counterparts. These features make them well suited for numerous applications such as quantum imaging or the generation of broadband squeezed light. In this context, some spatial aspects of TW-SHG were recently addressed in the framework of an experimental study [41], and although this work was carried out on a pure classical level, a detailed characterization of the spatial quantum properties of this system, including the spatial distribution of spontaneous emission appears to be relevant. The second motivation is of conceptual order: due to its simplicity, TW-SHG allows us to develop a clear physical picture of the origin of the diverse quantum correlations displayed by the fields, which might be useful for a better understanding of the general consequences of multimode entanglement.

This paper is organized as follows. In Sec. II, the physical system under study will be briefly presented, the phenomenon of spontaneous emission defined and the underlying microscopic processes identified. These considerations will provide a basis for the construction of a minimal four-mode model, which will be analyzed on a quantum level in Sec. III. The quantum correlations present in this model constitute

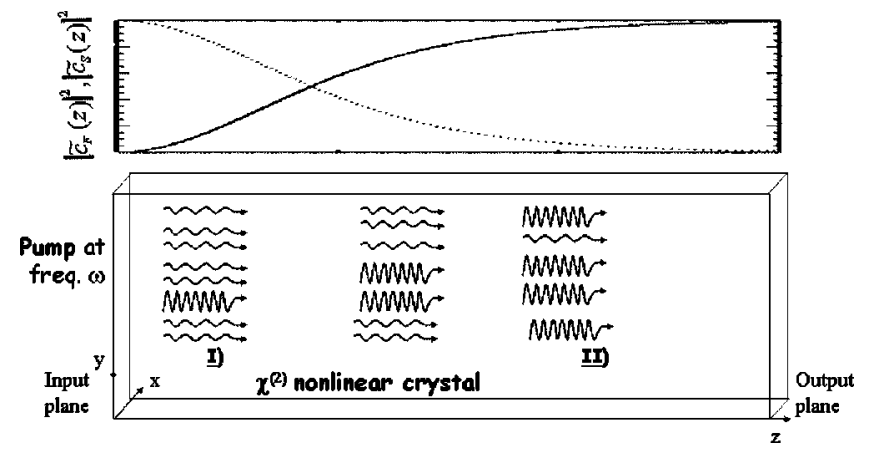

FIG. 1. SHG in the perfect phase-matched case. Upper part: The intensity of fundamental (dotted line) and SH field (solid line) in the crystal as a function of the propagation length. Lower part: Schematic representation of the different regions of the crystal. The horizontal axis $z$ corresponds to the direction of beam propagation, whereas $(x, y)$ defines the transverse plane. The slightly (strongly) wavy arrows represent fundamental $(\mathrm{SH})$ photons.

a valuable guideline for the understanding of the spatial correlations of the far-field intensity fluctuations in TW-SHG. The theoretical framework, based on the quantum description of the dynamics of the coupled system of fundamental and SH field in TW-SHG derived in Ref. [42], will be briefly summarized (Sec. IV). By linearizing the full nonlinear quantum propagation equations around the solution of classical nonlinear optics, we will show that quantum field propagation in TW-SHG can be described in terms of a fourmode dynamics, which couples pairs of field modes with opposite transverse wave vectors and frequency offsets at fundamental and second-harmonic frequency. As in the cavity counterpart of this system [40], this coupling results in interesting quantum correlations, including twin-beam correlations at both frequencies and cross correlations between fields at different frequencies. Expressions for all correlations of interest will be derived (Sec. V) and the results, obtained from a numerical integration of the propagation equations, presented and discussed in Sec. VI.

\section{THE MODEL}

The physical system we consider is a $\chi^{(2)}$ nonlinear crystal in the traveling-wave configuration, pumped with a monochromatic light source at frequency $\omega$. For simplicity, the pump intensity will be taken to be uniform in the transverse plane. Because of the quadratic nonlinearity of the crystal, a second-harmonic wave will be generated from the pump field and, in the general case, classical nonlinear optics predict a periodic transfer of energy between fundamental and $\mathrm{SH}$ field along the propagation axis [43]. In the particular case of a perfect phase matching [i.e., if $2 k_{F}-k_{S}=0$, where $k_{F}\left(k_{S}\right)$ is the wave number of a wave at frequency $\omega$ $(2 \omega)$ propagating in the NL crystal], the spatial period of these oscillations diverges and the efficiency of the frequency up-conversion of the pump increases monotonically as a function of the propagation length (Fig. 1). However, when the quantum fluctuations of the fields are taken into account, this process, at some propagation length, is reversed and the fundamental field starts being regenerated through 
down-conversion of the SH field [44]. In the following, we will assume that the length of the crystal is much smaller than this typical length, so that the complication connected with this "revival" can be disregarded.

In Ref. [42], this system has been studied in the context of quantum image processing, i.e., under the assumption that, in addition to the pump field, a coherent signal, encoding the image to be processed, was injected into the NL crystal. It has been pointed out that the main peculiarity of TW-SHG, with respect to the OPA pumped at second-harmonic frequency, relies on the presence of two "strong" homogeneous waves inside the crystal: the pump field at frequency $\omega$ and the SH field at twice this frequency, generated through SHG. As in an OPA, the latter can decay into pairs of counterpropagating fundamental photons and perform phasedependent amplification or deamplification of a signal at fundamental frequency, whereas the strong fundamental wave was shown to allow for the conversion of a signal from one frequency (fundamental or $\mathrm{SH}$ ) to the other.

Spontaneous emission, on which we focus in this paper, takes place without any coherent input signal, and is the direct manifestation of the vacuum quantum fluctuations which, as stated by quantum mechanics, enter the crystal at all temporal and spatial frequencies. As a result, the strong homogeneous SH wave can decay "spontaneously" into pairs of perfectly correlated twin photons,

$$
[2 \omega](\overrightarrow{0}, 0) \rightarrow[\omega](\vec{q}, \Omega)+[\omega](-\vec{q},-\Omega),
$$

with opposite transverse wave vectors and conjugate with respect to the fundamental frequency $\omega$, as a consequence of momentum, resp. energy conservation $([\omega](\vec{q}, \Omega)$ refers to a photon at frequency $\omega+\Omega$ and transverse wave vector $\vec{q}$, while $[2 \omega](\overrightarrow{0}, 0)$ represents an on-axis photon associated with the static homogeneous SH wave generated by SHG [45]). In the remaining of this paper, we will only consider the properties of the outgoing fields at nonvanishing wave vectors $\vec{q} \neq \overrightarrow{0}$, for which process (1) is a spontaneous emission process, since the modes $[\omega]( \pm \vec{q}, \pm \Omega)$ are originally in the vacuum state.

Further, due to the presence of the (depleted) fundamental pump field, some of the twin photons produced through Eq. (1) will be up-converted, according to the frequencychanging process:

$$
[\omega]( \pm \vec{q}, \pm \Omega)+[\omega](\overrightarrow{0}, 0) \rightarrow[2 \omega]( \pm \vec{q}, \pm \Omega) .
$$

Therefore, a fluorescence field at SH frequency will be generated as well (Fig. 2). The role of the two processes (1) and (2) in the stability of plane-wave pump beams propagating through $\chi^{(2)}$ NL media has been studied, on a classical level, in the temporal domain [46] and, recently, under consideration of the transverse spatial degrees of freedom [47]. At the level of a quantum description, process (2), absent in an OPA, will act randomly on some of the fundamental photons generated by process (1). Whereas the output field of an OPA exclusively consists of pairs of twin photons, what translates into perfect correlations of the fluctuations of the fundamen-

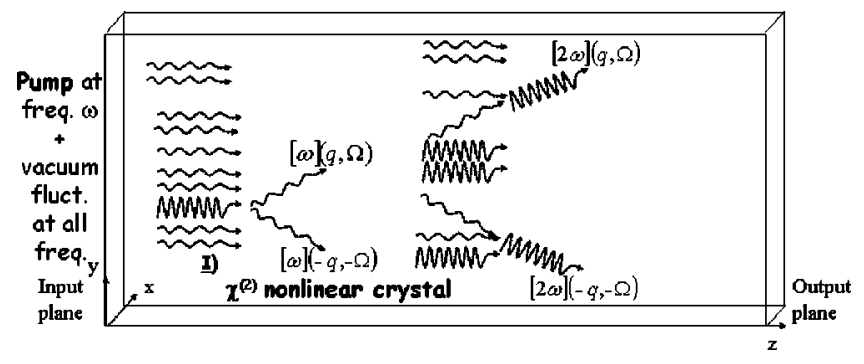

FIG. 2. Elementary processes, which are responsible for fluorescence in TW-SHG.

tal far-field intensities collected symmetrically with respect to the optical axis [48], process (2) will be equivalent to a loss mechanism. Therefore it is expected to lead to a degradation of the correlations generated by twin-photon emission. But simultaneously, as will be demonstrated in the rest of this paper, some twin-beam features will appear in the fluorescence field at SH frequency, as well as further correlations between fundamental and SH field. This is best demonstrated by the simple few-mode model developed in the following section.

\section{A SIMPLE FEW-MODE MODEL FOR TW-SHG}

Before considering a full multimodal description of the quantum field propagation in TW-SHG, it can be useful to construct a minimal quantum model which includes, in the simplest possible way, the elementary processes discussed in the preceding section, i.e., twin-photon production (1) and frequency up-conversion (2), acting separately on the twin photons. This model is represented in Fig. 3. The first part is an OPA, which generates pairs of tilted waves with opposite transverse wave vectors. For simplicity, we will only consider one pair of transverse modes $\omega_{+\vec{q}}$ and $\omega_{-\vec{q}}$ with wave vectors $\vec{q}$ and $-\vec{q}$. In a quantum description, the OPA is characterized by the following input-output transformations:

$$
\begin{aligned}
& \hat{b}_{F,+\vec{q}}=u \hat{a}_{F,+\vec{q}}+v \hat{a}_{F,-\vec{q}}^{\dagger}, \\
& \hat{b}_{F,-\vec{q}}=u \hat{a}_{F,-\vec{q}}+v \hat{a}_{F,+\vec{q}}^{\dagger}
\end{aligned}
$$

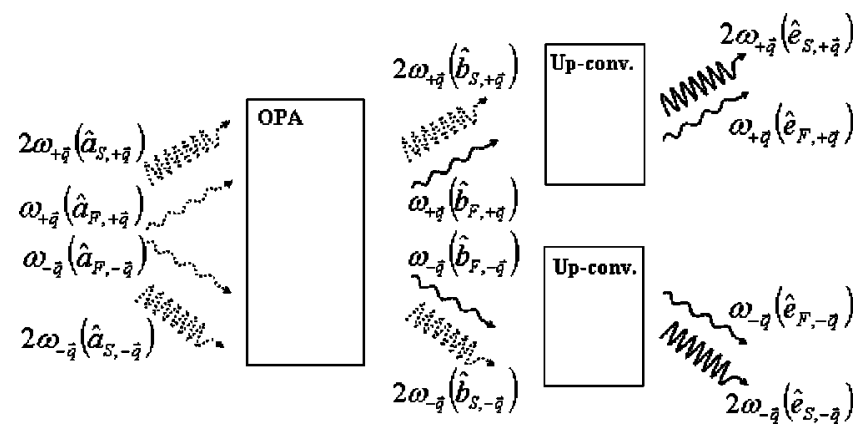

FIG. 3. Simplified model for spontaneous emission in TW-SHG: two counterpropagating waves generated by an OPA are separately injected into independent up-converters. The dotted waves represent the modes which are in the vacuum state. 

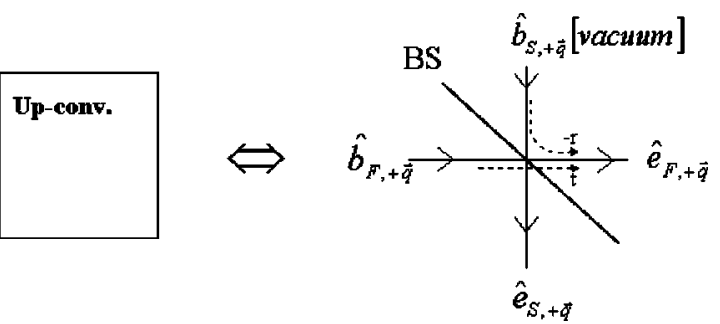

FIG. 4. Equivalence between up-conversion and a beam splitter.

where $\hat{b}_{F, \pm \vec{q}}\left(\hat{a}_{F, \pm \vec{q}}\right)$ are the operators associated with the two considered modes in the output (input) plane of the OPA. The standard commutation relations

$$
\left[\hat{b}_{F, \pm \vec{q}}, \hat{b}_{F, \pm \vec{q}}^{\dagger}\right]=\left[\hat{a}_{F, \pm \vec{q}}, \hat{a}_{F, \pm \vec{q}}^{\dagger}\right]=1
$$

impose the unitarity condition

$$
|u|^{2}-|v|^{2}=1
$$

The up-conversion process (2) will be modeled by assuming that each wave generated by the OPA is injected separately into a frequency up-converter. In a quantum description, frequency conversion, in the parametric approximation (i.e., under the assumption of a strong undepleted pump), is formally equivalent to a beam splitter (BS) [49,50]. Figure 4 explains this correspondence: one input port of the BS receives the incoming wave at fundamental frequency. This wave comes out with some attenuation at one of the output ports of the $\mathrm{BS}$, whereas the other output is identified with the fraction of the input, which has been up-converted to $\mathrm{SH}$ frequency. To preserve the commutation relations of all output fields, the remaining input port of the BS has to be formally illuminated with the vacuum fluctuations entering at SH frequency.

In terms of the quantum operators associated with the involved waves $\omega_{\vec{q}}$ and $2 \omega_{\vec{q}}$ (the notations can be obtained from Figs. 3 and 4), up-conversion is therefore expressed by the relations [51].

$$
\begin{gathered}
\hat{e}_{F, \vec{q}}=t \hat{b}_{F, \vec{q}}-r \hat{b}_{S, \vec{q}}, \\
\hat{e}_{S, \vec{q}}=r \hat{b}_{F, \vec{q}}+t \hat{b}_{S, \vec{q}}
\end{gathered}
$$

with the following condition for the coefficients $t$ and $r$, taken as real quantities:

$$
r^{2}+t^{2}=1
$$

For symmetry reasons, the operators associated with the waves with wave vector $-\vec{q}$ fulfill the same relations (6a) and (6b). Combining Eqs. (6) with Eqs. (3), we finally obtain the input-output transformations for the simple model represented in Fig. 3:

$$
\begin{aligned}
& \hat{e}_{F,+\vec{q}}=t u \hat{a}_{F,+\vec{q}}+t v \hat{a}_{F,-\vec{q}}^{\dagger}-r \hat{a}_{S,+\vec{q}}, \\
& \hat{e}_{S,+\vec{q}}=r u \hat{a}_{F,+\vec{q}}+r v \hat{a}_{F,-\vec{q}}^{\dagger}+t \hat{a}_{S,+\vec{q}},
\end{aligned}
$$

$$
\begin{aligned}
& \hat{e}_{F,-\vec{q}}=t v \hat{a}_{F,+\vec{q}}^{\dagger}+t u \hat{a}_{F,-\vec{q}}-r \hat{a}_{S,-\vec{q}}, \\
& \hat{e}_{S,+\vec{q}}=r v \hat{a}_{F,+\vec{q}}^{\dagger}+r u \hat{a}_{F,-\vec{q}}+t \hat{a}_{S,-\vec{q}} .
\end{aligned}
$$

Once the state of the incoming radiation is defined, which is here the vacuum state since we are interested in spontaneous emission, Eqs. (8) encode the full quantum statistics of the four coupled modes $\omega_{ \pm \vec{q}}$ and $2 \omega_{ \pm \vec{q}}$. In particular, this model predicts that the fluctuations of the photon numbers in the four modes are correlated. We first look for twin-beam correlations, i.e., nonclassical correlations of the photon number fluctuations in two counterpropagating modes. Introducing the operators associated with the photon number in each output mode $\hat{N}_{i, \pm \vec{q}}=\hat{e}_{i, \pm \vec{q}}^{\dagger} \hat{e}_{i, \pm \vec{q}}$, with $i=F, S$, the quantity of interest is the variance of the difference $\hat{N}_{i, \vec{q}}-\hat{N}_{i,-\vec{q}}$, normalized to the shot noise $\left(\mathcal{Z}_{S N}\right)$,

$$
V_{i i}^{(-)}[\vec{q},-\vec{q}]=\frac{\left\langle\left(\hat{N}_{i, \vec{q}}-\hat{N}_{i,-\vec{q}}\right)^{2}\right\rangle}{\mathcal{Z}_{S N}}
$$

which may drop below the classical limit of 1 [Eq. (9) already takes into account the symmetry $\left.\left\langle\hat{N}_{i, \vec{q}}\right\rangle=\left\langle\hat{N}_{i,-\vec{q}}\right\rangle\right]$. Inserting Eq. (8) into Eq. (9), one finds after some algebra

$$
\begin{aligned}
& V_{F F}^{(-)}[\vec{q},-\vec{q}]=\frac{\left\langle\left(\hat{N}_{F, \vec{q}}-\hat{N}_{F,-\vec{q}}\right)^{2}\right\rangle}{\mathcal{Z}_{S N}}=r^{2}, \\
& V_{S S}^{(-)}[\vec{q},-\vec{q}]=\frac{\left\langle\left(\hat{N}_{S, \vec{q}}-\hat{N}_{S,-\vec{q}}\right)^{2}\right\rangle}{\mathcal{Z}_{S N}}=t^{2} .
\end{aligned}
$$

These expressions, interestingly, do not depend on the parameters characterizing the OPA but only on those associated with the BS. The limit of a perfectly transmitting beam splitter $(t=1$ and hence $r=0)$ corresponds to no up-conversion acting on the OPA output and, as expected, Eq. (10a) reduces to $V_{F F}^{(-)}[\vec{q},-\vec{q}]=0$ : the correlations are perfect. In the opposite case $r=1$, the twin beams generated by the OPA are entirely up-converted to $\mathrm{SH}$ frequency, and hence the perfect twin-beam correlations are completely transferred to $\mathrm{SH}$ frequency $\left(V_{S S}^{(-)}[\vec{q},-\vec{q}]=0\right)$, whereas the difference $\hat{N}_{F, \vec{q}}$ $-\hat{N}_{F,-\vec{q}}$ is left with fluctuations at the shot noise level. These two limits are very similar to what occurs in intracavity SHG for very different losses at the two frequencies [52]: perfect squeezing is displayed by the fundamental field, if the cavity losses at SH frequency are insignificant (this corresponds to an up-conversion rate close to zero for the output fields), whereas in the opposite case of very low losses at fundamental frequency, the squeezing moves to the SH field. This phenomenon has been also recently discussed in the context of a quantum optical dimer [53].

An intermediary value of $r$ describes a partial upconversion of the twin beams generated by the OPA, with a rate equal to $r^{2}$. In this case, the variance $V_{F F}^{(-)}[\vec{q},-\vec{q}]$ does not vanish any more since the up-conversion mechanism randomly breaks pairs of twin photons by up-converting one of 
the two photons. The noise level in the photon number difference $\hat{N}_{F,+\vec{q}}-\hat{N}_{F,-\vec{q}}$ turns out to precisely coincide with the up-conversion rate:

$$
V_{F F}^{(-)}[\vec{q},-\vec{q}]=\frac{\left\langle\hat{N}_{S, \vec{q}}\right\rangle}{\left\langle\hat{N}_{F, \vec{q}}\right\rangle+\left\langle\hat{N}_{S, \vec{q}}\right\rangle} .
$$

As already mentioned, this twin-photon pair breaking process is not purely detrimental, since it induces some degree of twin-beam correlations at SH frequency: as can be seen from Eq. (10b), $V_{S S}^{(-)}[\vec{q},-\vec{q}]<1$. In this simple model, one observes that the twin-beam correlations at fundamental and SH frequency always sum up to 1 :

$$
V_{F F}^{(-)}[\vec{q},-\vec{q}]+V_{S S}^{(-)}[\vec{q},-\vec{q}]=1 .
$$

These elementary considerations demonstrate that twin-beam correlations do not always imply the existence of a microscopic process leading to the pairwise production of the photons of each beam. A secondary process acting separately on each of the two twin beams is enough to generate beams with intensity fluctuations, which are correlated to better than the shot noise.

Other interesting correlations are present in this simple four-mode model. The normalized variances

$$
V_{F S}^{(-)}[\vec{q}, \pm \vec{q}]=\frac{\left\langle\left(\hat{N}_{F, \vec{q}}-\hat{N}_{S, \pm q}\right)^{2}\right\rangle-\left\langle\left(\hat{N}_{F, \vec{q}}-\hat{N}_{S, \pm q}\right)\right\rangle^{2}}{\mathcal{Z}_{S N}}
$$

characterize the correlations between the fluctuations of the photon numbers in a fundamental and $\mathrm{SH}$ mode, either at the same $(+)$ or at opposite wave vectors $(-)$. Again, a simple calculation gives

$$
\begin{gathered}
V_{F S}^{(-)}[\vec{q}, \vec{q}]=1+v^{2}\left(t^{2}-r^{2}\right)^{2}, \\
V_{F S}^{(-)}[\vec{q},-\vec{q}]=1+v^{2}\left(t^{2}-r^{2}\right)^{2}-2 t^{2} r^{2} .
\end{gathered}
$$

These expressions deserve some comments. First considering the particular case of a balanced 50/50 beam splitter $(r=t$ $=1 / \sqrt{2}$ ), one ends up with the following equalities:

$$
V_{F F}^{(-)}[\vec{q},-\vec{q}]=V_{S S}^{(-)}[\vec{q},-\vec{q}]=V_{F S}^{(-)}[\vec{q},-\vec{q}]=\frac{1}{2},
$$

which indeed are not surprising, since a balanced 50/50 BS distributes randomly and with a probability $1 / 2$ the incoming photons over the two outputs. Hence, any output of one BS is equally correlated to any of the two outputs of the other BS. With respect to the perfectly correlated inputs of the two BS, this correlation is of course weaker but still nonclassical, since it corresponds to half the shot-noise level in the difference of the considered photon numbers. If we now consider the noise in the difference of the two outputs of a given BS with $r=t=1 / \sqrt{2}$, we find the standard quantum limit $V_{F S}^{(-)}[q, q]=1$. This result is also a consequence of the random character of the transmission or reflection process in a BS and is known to be independent of the photon statistics of the input beam [54]. In the general case (i.e., for an arbitrary value of $r), V_{F S}^{(-)}[q, q]$ and $V_{F S}^{(-)}[q,-q]$ do depend on the parameter $v$ characterizing the OPA. Inspecting Eqs. (14a) and (14b), one observes that

$$
V_{F S}^{(-)}[\vec{q},-\vec{q}]<V_{F S}^{(-)}[\vec{q}, \vec{q}] .
$$

At first glance, this result looks paradoxical, since it states that the photon number fluctuations at fundamental and SH frequency are more correlated at opposite wave vectors than at the same wave vector. The same counterintuitive behavior had been reported in Ref. [40] in the case of type-I intracavity SHG. The equivalence of up-conversion with a BS helps to elucidate this phenomenon. From Fig. 3, it is clear that the difference $\hat{N}_{F,+\vec{q}}-\hat{N}_{S,+\vec{q}}$ is not affected by the fact that the beam injected into the up-converter is one of the two perfectly correlated twin beams. On the contrary, the quantity $\hat{N}_{F,+\vec{q}}-\hat{N}_{S,-\vec{q}}$ keeps track of the twin-photon emission taking place in the OPA: as a matter of fact, since the inputs of both BS are perfectly correlated, there is a finite probability that an outgoing fundamental photon at $+\vec{q}$ is accompanied by a perfectly correlated SH photon at $-\vec{q}$ : this occurs each time the photon at $+\vec{q}$ of a given pair produced by the OPA is transmitted while the second at $-\vec{q}$ is up-converted. Evaluating the noise in $\hat{N}_{F,+\vec{q}}-\hat{N}_{S,-q}$, the two photons of the original twin-photon pair are recombined, and this leads to a partial noise cancellation. Comparing the noise level in $\hat{N}_{F,+\vec{q}}-\hat{N}_{S,+\vec{q}}$ to the one in $\hat{N}_{F,+\vec{q}}-\hat{N}_{S,-\vec{q}}$, one finds a noise reduction of $-2 t^{2} r^{2}$. This quantity can be understood by considering a twin-photon gun emitting pairs of photons at regular intervals. For a given shot, the photon at $+\vec{q}$ may either leave the system as a fundamental photon (with the probability amplitude $t$ ), giving rise to the output photon numbers $N_{F, q}=1$ and $N_{S, q}=0$, or be up-converted, and in this case, $N_{F, q}=0$ and $N_{S, q}=1$. The same occurs independently at the other BS placed at $-q$. The variances of interest can be written as

$$
\begin{aligned}
& \left\langle\left(\hat{N}_{F,+\vec{q}}-\hat{N}_{S,+\vec{q}}\right)^{2}\right\rangle-\left\langle\hat{N}_{F,+\vec{q}}-\hat{N}_{S,+\vec{q}}\right\rangle^{2} \\
& =\cdots-2\left\langle\hat{N}_{F,+\vec{q}} \cdot \hat{N}_{S,+\vec{q}},\right\rangle, \\
& \left\langle\left(\hat{N}_{F,+\vec{q}}-\hat{N}_{S,-\vec{q}}\right)^{2}\right\rangle-\left\langle\hat{N}_{F,+\vec{q}}-\hat{N}_{S,-\vec{q}}\right\rangle^{2} \\
& =\cdots-2\left\langle\hat{N}_{F,+\vec{q}} \cdot \hat{N}_{S,-\vec{q}}\right\rangle,
\end{aligned}
$$

where $\cdots$ stands for the common contributions to the two variances. The two $\mathrm{BS}$ being independent, $\left\langle N_{F,+\vec{q}} \cdot N_{S,-\vec{q}}\right\rangle$ on the right-hand side of Eq. (17b) can be evaluated by replacing the average of the product by the product of averages $t^{2} r^{2}$. On the other hand, the product $\left\langle N_{F,+\vec{q}} \cdot N_{S,+\vec{q}}\right\rangle$ is always zero because of photon number conservation. We obtain, as the difference between the two correlations, the result $-2 t^{2} r^{2}$. Finally, we note that whereas the difference $N_{F,+\vec{q}}$ $-N_{S, \vec{q}}$ is always above shot noise (or at shot noise if the up-conversion rate is $1 / 2$ ), the statistics of $N_{F,+\vec{q}}-N_{S,-\vec{q}}$ can be below this limit. This occurs if the parameter $v$, char- 
acterizing the OPA and in particular the amount of excess noise present in the individual beam injected into each upconverter, does not exceed the value $2 t^{2} r^{2} /\left(t^{2}-r^{2}\right)^{2}$.

Now, thinking in terms of the original problem of TWSHG, two aspects are missing in this simple model: first, the fact that twin-photon emission and up-conversion take place in fact simultaneously at any point of the crystal, while the scheme represented in Fig. 3 is based on the spatial separation of these two processes. Furthermore, the multimodal nature of field dynamics in TW-SHG has been so far ignored, since a single temporal mode was taken into account. In spite of these restrictions, the study of the simplified model provides us with a valuable guideline for the detailed investigation of the correlations in the fluorescence spectrum of TWSHG, which will be the subject of the remaining of this paper.

\section{A COMPLETE MULTIMODE QUANTUM-MECHANICAL DESCRIPTION OF SHG}

A quantum-mechanical description of the dynamics of the system of fundamental and SH field coupled through the $\chi^{(2)}$ nonlinearity of the crystal has been derived in Ref. [42]. In this section, we briefly review some general properties of this system and define some useful notation well suited for the study of a four-mode system. One first introduces the amplitude envelope operators $A_{F}(z, \vec{x}, t)$ and $A_{S}(z, \vec{x}, t)$ associated with fundamental and $\mathrm{SH}$ field, normalized in such a way that $A_{F}^{\dagger}(z, \vec{x}, t) A_{F}(z, \vec{x}, t)$ and $A_{S}^{\dagger}(z, \vec{x}, t) A_{S}(z, \vec{x}, t)$ coincide with the photon flux densities in photons per $\mathrm{cm}^{2}$ per sec at a point $\vec{x}$ on the transverse plane and at time $t$. $z$ is the coordinate on the beam propagation axis. These operators fulfill the free field commutation relation:

$$
\left[A_{i}(z, \vec{x}, t), A_{j}^{\dagger}\left(z, \vec{x}^{\prime}, t^{\prime}\right)\right]=\delta_{i j} \delta\left(\vec{x}-\vec{x}^{\prime}\right) \delta\left(t-t^{\prime}\right) .
$$

The propagation of these quantum fields in a $\chi^{(2)}$ nonlinear crystal was shown to be described by the following coupled equations [42]:

$$
\begin{aligned}
\frac{\partial}{\partial z} \hat{A}_{F}(z, \vec{q}, \Omega)= & -2 K \int d^{2} q^{\prime} d \Omega^{\prime} \hat{A}_{F}^{\dagger}\left(z, \vec{q}^{\prime}, \Omega^{\prime}\right) \\
& \times \hat{A}_{S}\left(z, \vec{q}+\vec{q}^{\prime}, \Omega+\Omega^{\prime}\right) \\
& \times e^{i\left(k_{S}^{z}\left(\vec{q}+\vec{q}^{\prime}, \Omega+\Omega^{\prime}\right)-k_{F}^{z}(\vec{q}, \Omega)-k_{F}^{z}\left(\vec{q}^{\prime}, \Omega^{\prime}\right)\right) z}
\end{aligned}
$$

$$
\begin{aligned}
\frac{\partial}{\partial z} \hat{A}_{S}(z, \vec{q}, \Omega)= & +K \int d^{2} q^{\prime} d \Omega^{\prime} \hat{A}_{F}\left(z, \vec{q}^{\prime}, \Omega^{\prime}\right) \\
& \times \hat{A}_{F}\left(z, \vec{q}-\vec{q}^{\prime}, \Omega-\Omega^{\prime}\right) \\
& \times e^{i\left(k_{F}^{z}\left(\vec{q}^{\prime}, \Omega^{\prime}\right)+k_{F}^{z}\left(\vec{q}-\vec{q}^{\prime}, \Omega-\Omega^{\prime}\right)-k_{S}^{z}(\vec{q}, \Omega)\right) z}
\end{aligned}
$$

where $\hat{A}_{i}(z, \vec{q}, \Omega)$ for $i=F, S$ is defined through the Fourier transform,

$$
\hat{A}_{i}(z, \vec{q}, \Omega)=e^{-i\left(k_{i}^{z}(\vec{q}, \Omega)-k_{i}\right) z} \int d^{2} x e^{-i \vec{q} \cdot \vec{x}} \int d t e^{i \Omega t} A_{i}(z, \vec{x}, t)
$$

and represents the propagation corrected annihilation operator of a photon with transverse wave vector $\vec{q}$, frequency $\omega_{i}+\Omega,(\Omega$ is the frequency offset with respect to the carrier frequency), and a longitudinal wave number deduced from the dispersion relation: $k_{i}^{z}(\vec{q}, \Omega)=\sqrt{k\left(\omega_{i}+\Omega\right)^{2}-\vec{q}^{2}}, k\left(\omega_{i}\right.$ $+\Omega$ ) giving the wave number of a wave with frequency $\omega_{i}+\Omega$ propagating in the NL crystal under consideration. In particular, the wave numbers $k_{i}$ introduced in Eq. (20) are $k_{i}=k\left(\omega_{i}\right) . K$ is the coupling constant of the nonlinear interaction and is proportional to the nonlinear susceptibility $\chi^{(2)}$. To solve Eqs. (19a) and (19b), one usually separates the strong homogeneous fields created by the pump inside the crystal by means of the ansatz,

$$
\begin{aligned}
& \hat{A}_{F}(z, \vec{q}, \Omega)=c_{F}(z) \delta^{(2)}(\vec{q}) \delta(\Omega)+\hat{a}_{F}(z, \vec{q}, \Omega), \\
& \hat{A}_{S}(z, \vec{q}, \Omega)=c_{S}(z) \delta^{(2)}(\vec{q}) \delta(\Omega)+\hat{a}_{S}(z, \vec{q}, \Omega),
\end{aligned}
$$

in which $c_{F}(z)$ and $c_{S}(z)$ are the amplitudes of these strong monochromatic waves at frequencies $\omega$ and $2 \omega$. The product of $\delta$-functions encodes the assumption of a stationaries and transversally homogeneous pump. In the small fluctuation approximation, a system of two linear coupled propagation equations for the quantum operators associated with fundamental and SH field is obtained [42]:

$$
\begin{aligned}
\frac{\partial}{\partial \tilde{z}} \hat{a}_{F}(\tilde{z}, \vec{q}, \Omega)= & -\tilde{c}_{S}(\tilde{z}) \hat{a}_{F}^{\dagger}(\tilde{z},-\vec{q},-\Omega) e^{-i \widetilde{\Delta}(\vec{q}, \Omega) \tilde{z}} \\
& -\sqrt{2} \tilde{c}_{F}^{*}(\tilde{z}) \hat{a}_{S}(\tilde{z}, \vec{q}, \Omega) e^{-i \tilde{D}(\vec{q}, \Omega) \tilde{z}}, \\
\frac{\partial}{\partial \tilde{z}} \hat{a}_{S}(\tilde{z}, \vec{q}, \Omega)= & +\sqrt{2} \tilde{c}_{F}(\tilde{z}) \hat{a}_{F}(\tilde{z}, \vec{q}, \Omega) e^{i \tilde{D}(\vec{q}, \Omega) \tilde{z}}
\end{aligned}
$$

whereas the normalized amplitudes $\tilde{c}_{F}(\tilde{z})=c_{F}(z) / \sqrt{W}$ and $\tilde{c}_{S}(\tilde{z})=c_{S}(z) / \sqrt{W / 2}$, with $W=\left|c_{F}(0)\right|^{2}+2\left|c_{S}(0)\right|^{2}$ defined as the input power injected into the nonlinear crystal, satisfy, as expected, the classical equations of nonlinear optics:

$$
\begin{gathered}
\frac{d}{d \tilde{z}} \tilde{c}_{F}(\tilde{z})=-\tilde{c}_{F}^{*}(\tilde{z}) \tilde{c}_{S}(\tilde{z}) e^{-i \Delta s \tilde{z}}, \\
\frac{d}{d \tilde{z}} \tilde{c}_{S}(\tilde{z})=+\tilde{c}_{F}^{2}(\tilde{z}) e^{i \Delta s \tilde{z}} .
\end{gathered}
$$

The dimensionless interaction length $\tilde{z}$ is the propagation length expressed in units of $z_{0}=1 /(\sqrt{2 W} K)$, the characteristic length scale in phase-matched SHG. $\Delta s=\left(2 k_{F}-k_{S}\right) z_{0}$ represents the dimensionless collinear phase mismatch. In the case of a vanishing phase mismatch $\Delta s=0$, the solution of Eqs. (23) is particularly simple and reads [43] 


$$
\begin{aligned}
& \tilde{c}_{F}(\tilde{z})=e^{i \phi_{F}^{(0)}} \operatorname{sech}(\tilde{z}), \\
& \tilde{c}_{S}(\tilde{z})=e^{2 i \phi_{F}^{(0)}} \tanh (\tilde{z}),
\end{aligned}
$$

where $\phi_{F}^{(0)}$ is the phase of the pump field at the input plane of the crystal, which, in the context of spontaneous emission, is irrelevant.

The propagation equations for the quantum field operators (22a) and (22b) clearly display the two types of elementary processes used in Sec. III to construct the simplified model. The first term on the rhs of Eq. (22a) couples the fundamental field amplitude $\hat{a}_{F}(\tilde{z}, \vec{q}, \Omega)$ to $\hat{a}_{F}^{\dagger}(\tilde{z},-\vec{q},-\Omega)$ and originates in the photon down-conversion (1) of the strong $\mathrm{SH}$ wave generated in the crystal through SHG. Its amplitude $\tilde{c}_{S}(\tilde{z})$, combined with a phase factor involving an effective phase mismatch along the beam propagation direction,

$$
\tilde{\Delta}(\vec{q}, \Omega)=\left(k_{F}^{z}(\vec{q}, \Omega)+k_{F}^{z}(-\vec{q},-\Omega)-k_{S}\right) z_{0}
$$

governs the efficiency of this process. The frequency changing process (2) generates a coupling of the amplitude $\hat{a}_{F}(\tilde{z}, \vec{q}, \Omega)$ to $\hat{a}_{S}(\tilde{z}, \vec{q}, \Omega)$, encoded through the second term on the rhs of Eq. (22a) and the rhs of Eq. (22b). The pump for this process is the depleted pump field $\tilde{c}_{F}(\tilde{z})=\operatorname{sech}(\tilde{z})$, which appears as a prefactor, as well as a complex exponential factor involving a second effective phase mismatch

$$
\widetilde{D}(\vec{q}, \Omega)=\left[k_{F}^{z}(\vec{q}, \Omega)+k_{F}-k_{S}^{z}(\vec{q}, \Omega)\right] z_{0},
$$

whose effect will be to limit the efficiency to a certain range of spatial and temporal frequencies. In the standard paraxial and monochromatic approximation, the effective phase mismatches,

$$
\begin{gathered}
\tilde{\Delta}(\vec{q}, \Omega)=\Delta s+\operatorname{sgn}\left(k_{F}^{\prime \prime}\right) \frac{\Omega^{2}}{\Omega_{2}^{2}}-\frac{q^{2}}{q_{2}^{2}}, \\
\widetilde{D}(\vec{q}, \Omega)=\Delta s-\frac{\Omega}{\Omega_{1}}+\operatorname{sgn}\left(k_{F}^{\prime \prime}\right) \frac{\Omega^{2}}{4 \Omega_{2}^{2}}-\frac{1}{4}\left(1-\frac{\Delta k}{2 k_{F}}\right) \frac{q^{2}}{q_{2}^{2}},
\end{gathered}
$$

involve one typical spatial frequency $q_{2}=\sqrt{k_{F} / z_{0}}$ and two distinct temporal frequencies $\Omega_{1}=\left[\left(2 n_{S}^{\prime}-n_{F}^{\prime}\right) z_{0} \omega / c\right]^{-1}$ and $\Omega_{2}=\left(\left|k_{F}^{\prime \prime}\right| z_{0}\right)^{-1 / 2}$, with $k_{F}^{\prime \prime}=k^{\prime \prime}(\omega)$ and $n_{i}^{\prime}=n^{\prime}\left(\omega_{i}\right), n\left(\omega_{i}\right)$ being the refraction index of the crystal at frequency $\omega_{i}$ $=\omega(i=F)$ or $2 \omega(i=S) . \Omega_{2}$ and $q_{2}$ are known from the theory of the OPA to set the bandwidths for amplification/ deamplification of an input signal and squeezing at fundamental frequency [55]. $\Omega_{1}$ comes in through the first-order dependence of $\widetilde{D}(\vec{q}, \Omega)$ on $\Omega$, and originates in the group velocity mismatch between fundamental and $\mathrm{SH}$ wave [56]. As can be seen from Eqs. (22a) and (22b), it determines the bandwidth of the coupling between $\mathrm{SH}$ and fundamental field [57]. Expressing $q$ and $\Omega$ in units of $q_{2}$ and $\Omega_{2}(\widetilde{\Omega}$ $\left.=\Omega / \Omega_{2}, \tilde{q}=q / q_{2}\right)$, and assuming that $\Delta k / 2 k_{F} \ll 1$, one ends up with the following expressions:

$$
\begin{gathered}
\widetilde{\Delta}(\vec{q}, \Omega)=\Delta s+{\widetilde{\Omega^{2}}}^{2} \widetilde{q}^{2}, \\
\widetilde{D}(\vec{q}, \Omega)=\Delta s-\eta \widetilde{\Omega}+\frac{\widetilde{\Omega}^{2}}{4}-\frac{\widetilde{q}^{2}}{4},
\end{gathered}
$$

with $\eta=\Omega_{2} / \Omega_{1}$. In all numerical calculations, we will set $\eta=4$, which is compatible with the value considered in Ref. [56]. In addition, we restrict our study to the normal dispersion regime, for which $\operatorname{sgn}\left(k_{F}^{\prime \prime}\right)=1$.

Integrating Eqs. (22a) and (22b), one can express the output field operators $\hat{e}_{i}(\vec{q}, \Omega)=\hat{a}_{i}\left(\tilde{z}_{L}, \vec{q}, \Omega\right)$ defined in the exit plane of the crystal ( $\tilde{z}_{L}$ being the length of the crystal in units of $\left.z_{0}\right)$ in terms of the operators $\hat{a}_{i}(\vec{q}, \Omega)=\hat{a}_{i}(0, \vec{q}, \Omega)$ associated with the fields in the input plane. One finally obtains the following input-output transformations for the system of fundamental and SH field in TW-SHG:

$$
\begin{aligned}
\hat{e}_{F}(\vec{q}, \Omega)= & u_{F}(\vec{q}, \Omega) \hat{a}_{F}(\vec{q}, \Omega)+v_{F}(\vec{q}, \Omega) \hat{a}_{F}^{\dagger}(-\vec{q},-\Omega) \\
& +\mu_{F}(\vec{q}, \Omega) \hat{a}_{S}(\vec{q}, \Omega)+\nu_{F}(\vec{q}, \Omega) \hat{a}_{S}^{\dagger}(-\vec{q},-\Omega)
\end{aligned}
$$

$$
\begin{aligned}
\hat{e}_{S}(\vec{q}, \Omega)= & u_{S}(\vec{q}, \Omega) \hat{a}_{F}(\vec{q}, \Omega)+v_{S}(\vec{q}, \Omega) \hat{a}_{F}^{\dagger}(-\vec{q},-\Omega) \\
& +\mu_{S}(\vec{q}, \Omega) \hat{a}_{S}(\vec{q}, \Omega)+\nu_{S}(\vec{q}, \Omega) \hat{a}_{S}^{\dagger}(-\vec{q},-\Omega),
\end{aligned}
$$

which generalizes to the case of two coupled fields, the input-output transformation

$$
\hat{e}_{F}(\vec{q}, \Omega)=u_{F}(\vec{q}, \Omega) \hat{a}_{F}(\vec{q}, \Omega)+v_{F}(\vec{q}, \Omega) \hat{a}_{F}^{\dagger}(-\vec{q},-\Omega)
$$

describing the propagation of fundamental field in an OPA [55]. Transformation (29) reflects the coupling of the four field modes associated with the waves $[\omega](q, \Omega),[\omega]$ $(-q,-\Omega),[2 \omega](q, \Omega),[2 \omega](-q,-\Omega)$ induced during propagation by the two microscopic processes (1) and (2). To avoid lengthy formulas, it is advantageous to introduce a vectorlike notation by defining the two-component operators $\vec{a}(\vec{q}, \Omega)$ and $\vec{a}^{\dagger}(\vec{q}, \Omega)$,

$$
\vec{a}(\vec{q}, \Omega)=\left(\begin{array}{c}
\hat{a}_{F}(\vec{q}, \Omega) \\
\hat{a}_{S}(\vec{q}, \Omega)
\end{array}\right), \quad \vec{a}^{\dagger}(\vec{q}, \Omega)=\left(\begin{array}{c}
\hat{a}_{F}^{\dagger}(\vec{q}, \Omega) \\
\hat{a}_{S}^{\dagger}(\vec{q}, \Omega)
\end{array}\right)
$$

and arranging the coefficients of Eqs. (29) as

$$
\vec{U}_{i}(\vec{q}, \Omega)=\left(\begin{array}{c}
u_{i}(\vec{q}, \Omega) \\
\mu_{i}(\vec{q}, \Omega)
\end{array}\right) \quad \vec{V}_{i}(\vec{q}, \Omega)=\left(\begin{array}{c}
v_{i}(\vec{q}, \Omega) \\
\nu_{i}(\vec{q}, \Omega),
\end{array}\right)
$$

with $i=F, S$. This allows us to rewrite the input-output transformation in the following compact way:

$$
\hat{e}_{F}(\vec{q}, \Omega)=\vec{U}_{F}(\vec{q}, \Omega) \cdot \vec{a}(\vec{q}, \Omega)+\vec{V}_{F}(\vec{q}, \Omega) \cdot \vec{a}^{\dagger}(-\vec{q},-\Omega),
$$




$$
\hat{e}_{S}(\vec{q}, \Omega)=\vec{U}_{S}(\vec{q}, \Omega) \cdot \vec{a}(\vec{q}, \Omega)+\vec{V}_{S}(\vec{q}, \Omega) \cdot \vec{a}^{\dagger}(-\vec{q},-\Omega) .
$$

The eight complex coefficients of the input-output transformation (29a) and (29b) are not independent. First, the outputfield operators, like the ones characterizing the input fields, have to satisfy the free-field commutation relations:

$$
\begin{aligned}
{\left[\hat{e}_{i}(\vec{q}, \Omega), \hat{e}_{j}^{\dagger}\left(\vec{q}^{\prime}, \Omega^{\prime}\right)\right] } & =\left[\hat{a}_{i}(\vec{q}, \Omega), \hat{a}_{j}^{\dagger}\left(\vec{q}^{\prime}, \Omega^{\prime}\right)\right] \\
& =(2 \pi)^{3} \delta^{(2)}\left(\vec{q}-\vec{q}^{\prime}\right) \delta\left(\Omega-\Omega^{\prime}\right) \delta_{i j} .
\end{aligned}
$$

This condition translates into the following relation for the coefficients:

$$
\vec{U}_{i}^{*}(\vec{q}, \Omega) \cdot \vec{U}_{j}(\vec{q}, \Omega)-\vec{V}_{i}^{*}(\vec{q}, \Omega) \cdot \vec{V}_{j}(\vec{q}, \Omega)=\delta_{i j} .
$$

From the requirement that operators associated with fields at different frequencies must commute $\left[\hat{e}_{i}(\vec{q}, \Omega), \hat{e}_{j}\left(\vec{q}^{\prime}, \Omega^{\prime}\right)\right]$ $=0$, one obtains a further condition

$$
\vec{U}_{i}(\vec{q}, \Omega) \cdot \vec{V}_{j}(-\vec{q},-\Omega)=\vec{U}_{j}(-\vec{q},-\Omega) \cdot \vec{V}_{i}(\vec{q}, \Omega) .
$$

It is worth mentioning that within this vectorial notation, Eqs. (35) and (36) for $i=j=F$ are formally identical to the relations fulfilled by the coefficients of the input-output transformation (30) for the OPA and used in Ref. [48]. Finally, from energy-momentum conservation, another relation can be derived:

$$
\begin{aligned}
\vec{V}_{F}^{*}(\vec{q}, \Omega) \cdot \vec{V}_{F}(\vec{q}, \Omega)+\vec{V}_{S}^{*}(\vec{q}, \Omega) \cdot \vec{V}_{S}(\vec{q}, \Omega) \\
=\vec{V}_{F}^{*}(-\vec{q},-\Omega) \cdot \vec{V}_{F}(-\vec{q},-\Omega) \\
\quad+\vec{V}_{S}^{*}(-\vec{q},-\Omega) \cdot \vec{V}_{S}(-\vec{q},-\Omega) .
\end{aligned}
$$

Analytical expressions for the coefficients of the two-field input-output transformation are available so far only for $q$ $=0$ and $\Omega=0$ [57]. In the case of arbitrary values of spatial and temporal frequency, no analytical solution of the propagation equations is known, and the results presented in Sec. VI were obtained by means of a numerical integration of Eqs. (22a) and (22b) [42].

\section{CORRELATIONS IN SECOND-HARMONIC GENERATION}

The general structure of the input-output transformation (29a) and (29b) indicates the presence of quantum correlations within any four-mode subsystem built by two fundamental modes with opposite transverse wave vectors $\vec{q}$ and $-\vec{q}$ and frequencies $\omega+\Omega$ and $\omega-\Omega$, and two SH modes at the same wave vectors and frequencies $2 \omega+\Omega$ and $2 \omega$ $-\Omega$. These correlations originate in the microscopic processes (1) and (2): the first correlates the modes $[\omega](q, \Omega)$ and $[\omega](-q,-\Omega)$ associated to a pair of twin photons, whereas the latter induces population exchanges between the

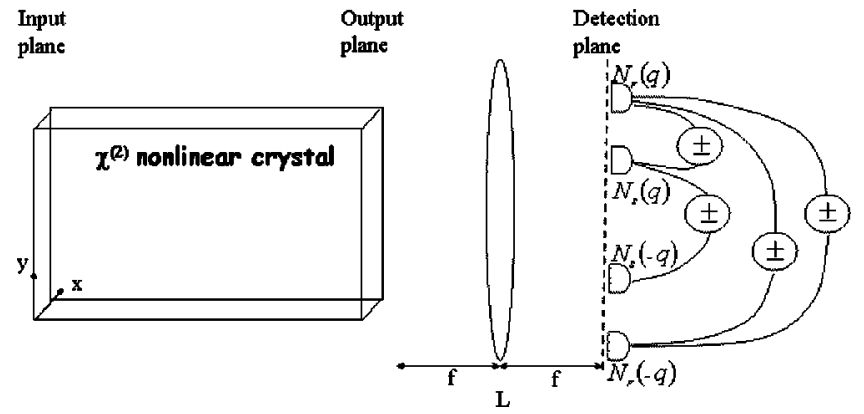

FIG. 5. Experimental setup for the investigation of spatial correlations in TW-SHG.

modes $[\omega](q, \Omega)$ and $[2 \omega](q, \Omega)$, resp. $[\omega](-q,-\Omega)$ and $[2 \omega](-q,-\Omega)$ via sum-frequency mixing with an on-axis pump photon $[\omega](0,0)[47,58]$. However, the strength of these correlations depends on $\vec{q}$ and $\Omega$ in a way which is essentially determined by the phase-mismatch functions $\widetilde{\Delta}(\vec{q}, \Omega)$ and $\widetilde{D}(\vec{q}, \Omega)$ [Eqs. (28a) and (28b)]. One manifestation of these quantum correlations is the existence of correlations in the fluctuations of the intensities at both frequencies measured in symmetrical portions of the far-field plane [59]. The experimental setup, which would allow us to measure these correlations, is sketched in Fig. 5.

The fluorescence field is imaged on the detection plane by means of a one-lens-imaging system. As stated by standard results of classical optics, the field on the detection plane $\mathcal{E}_{i}(\vec{x}, t)$ is the spatial Fourier transform of the output field $A_{i}\left(z_{L}, \vec{x}, t\right)$,

$$
\begin{aligned}
\mathcal{E}_{i}(\vec{x}, t) & =-\frac{i}{\lambda_{i} f} \int d^{2} x^{\prime} A_{i}\left(z_{L}, \vec{x}^{\prime}, t\right) e^{-i\left(2 \pi / \lambda_{i} f\right) \vec{x} \cdot \vec{x}^{\prime}} \\
& =-\frac{i}{\lambda_{i} f} \hat{e}_{i}\left(\frac{2 \pi}{\lambda_{i} f} \vec{x}, t\right)
\end{aligned}
$$

where $\hat{e}_{i}(\vec{k}, t)$ represents the quantum operator associated with an outgoing wave with frequency $\omega_{i}$ and wave vector $\vec{k}$ at time $t$. Therefore, the lens images an outgoing plane wave with transverse wave vector $\vec{q}$ at a point $\vec{x}_{i}=\left(f / k_{i}\right) \vec{q}$ of the detection plane, $f$ being the focal length of the lens. Since the position of this point depends on the wavelength under consideration, the far-field intensities at fundamental and $\mathrm{SH}$ frequency for a given wave vector can be detected in a single measurement. In the case of perfect phase matching, an array of four detectors placed at $\vec{x}_{F},-\vec{x}_{F}, \vec{x}_{F} / 2,-\vec{x}_{F} / 2$, with $\vec{x}_{F}$ $=\left(f / k_{F}\right) \vec{q}$, would allow us to measure simultaneously the intensity of fundamental and SH mode at transverse wave vectors $\vec{q}$ and $-\vec{q}$ and to determine experimentally the correlations of interest. Assuming for simplicity a perfect quantum efficiency of the detectors, the measured quantities can be expressed in terms of the operators associated with the number of outgoing photons:

$$
\hat{N}_{i}(q)=\int_{\sigma(q)} d^{2} k \int_{-\tau_{d} / 2}^{\tau_{d} / 2} d t \hat{e}_{i}^{\dagger}(k, t) \hat{e}_{i}(k, t) .
$$


The time integration accounts for a finite acquisition time $\tau_{d}$, and the size of the detector in real space is modeled by integrating the local intensity over a surface $\sigma(q)$ of area $\sigma_{d}$ centered on an average wave number $q$. Going to time frequencies, expression (39) reads

$$
\begin{aligned}
\hat{N}_{i}(q)= & \int_{\sigma(q)} d^{2} k \int \frac{d \Omega_{1} d \Omega_{2}}{(2 \pi)^{2}} \mathcal{F}\left(\Omega_{1}-\Omega_{2}\right) \\
& \times \hat{e}_{i}^{*}\left(k, \Omega_{1}\right) \hat{e}_{i}\left(k, \Omega_{2}\right),
\end{aligned}
$$

where the function

$$
\mathcal{F}(\Omega)=\int_{-\tau / 2}^{\tau / 2} d t e^{i \Omega t}=\frac{\sin (\Omega \tau)}{\Omega}
$$

encodes the information about the finite time measurement. The field operators involved in Eq. (40) are defined by Eqs. (29), while it is assumed that the detection area $\sigma(q)$ does not contain the direction of the optical axis $q=0$, so that the coherent field components do not impinge on the detector. The correlations of the intensity fluctuations are encoded in the following shot-noise normalized variances:

$$
\begin{aligned}
V_{i j}^{(\epsilon)}(q, \nu q) \\
\quad=\frac{\left\langle\left[\hat{N}_{i}(q)+\epsilon \hat{N}_{j}(\nu q)\right]^{2}\right\rangle-\left\langle\left[\hat{N}_{i}(q)+\epsilon \hat{N}_{j}(\nu q)\right]\right\rangle^{2}}{\mathcal{Z}_{S N}},
\end{aligned}
$$

with $i, j=F, S, \epsilon= \pm$ and $\nu= \pm$ (The notation $\langle\cdots\rangle$ denotes vacuum expectation values [60].) More specifically, correlations between counterpropagating modes of each field will be addressed by setting $i=j$, while cross correlations connecting modes of fields at different frequencies correspond to $i \neq j$. The parameter $\nu$ is +1 , if the far-field correlations at a given wave vector are investigated, or -1 at opposite wave vectors. Finally, $\epsilon=1$ corresponds to evaluating the noise level in the sum of the photon intensities, whereas in the case $\epsilon=-1$ the noise in the intensity difference will be computed. To express variances (42) in terms of the coefficients of input-output transformation (29), we first define the unnormalized correlation function:

$$
\begin{aligned}
C_{i j}^{\epsilon}(q, \nu q) & =\left\langle\left[\hat{N}_{i}(q)+\epsilon \hat{N}_{j}(\nu q)\right]^{2}\right\rangle-\left\langle\left[\hat{N}_{i}(q)+\epsilon \hat{N}_{j}(\nu q)\right]\right\rangle^{2} \\
& =\mathcal{X}_{i i}(q, q)+\mathcal{X}_{j j}(\nu q, \nu q)+2 \epsilon \mathcal{X}_{i j}(q, \nu q)
\end{aligned}
$$

where the photon number variance

$$
\mathcal{X}_{i j}(q, \nu q)=\left\langle\hat{N}_{i}(q) \hat{N}_{j}(\nu q)\right\rangle-\left\langle\hat{N}_{i}(q)\right\rangle\left\langle\hat{N}_{j}(\nu q)\right\rangle
$$

can be rewritten as

$$
\begin{aligned}
\mathcal{X}_{i j}(q, v q)= & \int_{\sigma(q)} d^{2} k \int_{\sigma(\nu q)} d^{2} k^{\prime} \int \frac{d \Omega_{1} d \Omega_{2}}{(2 \pi)^{2}} \int \frac{d \Omega_{3} d \Omega_{4}}{(2 \pi)^{2}} \\
& \times \mathcal{F}\left(\Omega_{1}-\Omega_{2}\right) \mathcal{F}\left(\Omega_{3}-\Omega_{4}\right) \\
& \times\left[\left\langle\hat{e}_{i}^{\dagger}\left(k, \Omega_{1}\right) \hat{e}_{i}\left(k, \Omega_{2}\right) \hat{e}_{j}^{\dagger}\left(k^{\prime}, \Omega_{3}\right) \hat{e}_{j}\left(k^{\prime}, \Omega_{4}\right)\right\rangle\right. \\
& \left.-\left\langle\hat{e}_{i}^{\dagger}\left(k, \Omega_{1}\right) \hat{e}_{i}\left(k, \Omega_{2}\right)\right\rangle\left\langle\hat{e}_{j}^{\dagger}\left(k^{\prime}, \Omega_{3}\right) \hat{e}_{j}\left(k^{\prime}, \Omega_{4}\right)\right\rangle\right] .
\end{aligned}
$$

Making use of the free-field commutation relations (34), expression (45) can be converted into a normal ordered expression, the extra terms corresponding to the associated shotnoise level:

$$
\begin{aligned}
\mathcal{Z}_{S N}\left[\mathcal{X}_{i j}(q, \nu q)\right]= & \delta_{\nu, 1} \delta_{i, j} \int_{\sigma(q)} d^{2} k \int \frac{d \Omega_{1} d \Omega_{2} d \Omega_{4}}{(2 \pi)^{4}} \\
& \times \mathcal{F}\left(\Omega_{1}-\Omega_{2}\right) \mathcal{F}\left(\Omega_{2}-\Omega_{4}\right) \\
& \times\left\langle\hat{e}_{i}^{\dagger}\left(k, \Omega_{1}\right) \hat{e}_{i}\left(k, \Omega_{4}\right)\right\rangle .
\end{aligned}
$$

Before inserting Eq. (33) into Eqs. (45) and (46), we make the following simplifying assumptions: first we assume that the acquisition time $\tau$ is much larger than the two time scales $\Omega_{2}^{-1}$ and $\Omega_{1}^{-1}$ associated with the two microscopic processes of photon down-conversion and up-conversion. Furthermore, we consider detectors which are small enough, so that the variations of the fields on the detection area can be neglected. After some bosonic operator algebra, we end up with the following expressions:

$$
\begin{aligned}
& \mathcal{X}_{i j}(q, \nu q)= \frac{\tau_{d} \sigma_{d}}{2 \pi^{3}}\left[\delta_{\nu, 1} \int d \Omega\left[\vec{V}_{i}^{*}(q, \Omega) \cdot \vec{V}_{j}(q, \Omega)\right]\right. \\
& \times\left[\vec{U}_{i}(q, \Omega) \cdot \vec{U}_{j}^{*}(q, \Omega)\right] \\
&\left.+\delta_{\nu,-1} \int d \Omega\left|\vec{U}_{i}(q, \Omega) \cdot \vec{V}_{j}(-q,-\Omega)\right|^{2}\right] \\
& \times \delta^{(2)}(\overrightarrow{0}), \\
& \mathcal{Z}_{S N}\left[\mathcal{X}_{i j}(q, \nu q)\right] \\
&=\frac{\tau_{d} \sigma_{d}}{2 \pi^{3}}\left[\delta_{\nu, 1} \delta_{i, j} \int d \Omega \vec{V}_{i}^{*}(q, \Omega) \cdot \vec{V}_{i}(q, \Omega)\right] \delta^{(2)}(\overrightarrow{0}) .
\end{aligned}
$$

The presence of the divergent factor $\delta^{(2)}(\overrightarrow{0})$ in both expressions can be traced back to the assumption of a crystal with infinite transverse dimensions. As shown in Ref. [61], a natural way to regularize this divergence consists of placing a pupil in the output plane of the nonlinear crystal. Under certain additional assumptions, this procedure merely amounts in replacing $\delta^{(2)}(\overrightarrow{0})$ by a factor $S_{p} / \lambda^{2} f^{2}$, depending on $S_{p}$, the area of the pupil. Defining the few following integrals, which will be evaluated numerically 


$$
\begin{gathered}
I_{i}(q)=\int d \Omega \vec{V}_{i}^{*}(q, \Omega) \cdot \vec{V}_{i}(q, \Omega) \\
J_{i}(q)=\int d \Omega\left[\vec{V}_{i}^{*}(q, \Omega) \cdot \vec{V}_{i}(q, \Omega)\right]\left[\vec{U}_{i}^{*}(q, \Omega) \cdot \vec{U}_{i}(q, \Omega)\right] \\
K_{i}(q)=\int d \Omega\left|\vec{U}_{i}(q, \Omega) \cdot \vec{V}_{i}(-q,-\Omega)\right|^{2} \\
L_{m}(q)=\int d \Omega\left|\vec{U}_{F}(q, \Omega) \cdot \vec{V}_{S}(-q,-\Omega)\right|^{2} \\
L_{p}(q)=\int d \Omega\left|\vec{V}_{F}^{*}(q, \Omega) \cdot \vec{V}_{S}(q, \Omega)\right|^{2}
\end{gathered}
$$

the variances of interest can be expressed as follows:

$$
\begin{gathered}
V_{i i}^{(+)}(q, q)=\frac{J_{i}(q)}{I_{i}(q)}, \\
V_{i i}^{(\epsilon)}(q,-q)=\frac{J_{i}(q)+\epsilon K_{i}(q)}{I_{i}(q)}, \\
V_{F S}^{(\epsilon)}(q, q)=\frac{J_{F}(q)+J_{S}(q)+2 \epsilon L_{m}(q)}{I_{F}(q)+I_{S}(q)}, \\
V_{F S}^{(\epsilon)}(q,-q)=\frac{J_{F}(q)+J_{S}(q)+2 \epsilon L_{p}(q)}{I_{F}(q)+I_{S}(q)} .
\end{gathered}
$$

Recalling definition (42), $V_{F F}^{(+)}(q, q)$ and $V_{S S}^{(+)}(q, q)$ characterize the statistics of the individual photocurrents at fundamental and SH frequency, $V_{i i}^{(-)}(q,-q)$ being the twin-beam correlations at each frequency, while $V_{F S}^{(\epsilon)}(q, \pm q)$ allows to detect the presence of correlations in the fluctuations of the intensities at the two frequencies either at the same wave vector $(+)$, or at opposite wave vectors $(-)$. Finally, it should be pointed out that these expressions were derived for nonvanishing transverse wave vectors. The special case of the correlations at zero transverse wave vector, for which the coherent component of the fields gives rise to additional contributions to the variances of interest, has been treated in Ref. [62] and will not be considered here.

\section{RESULTS}

We first investigate the distribution of fluorescence field in the transverse plane. The mean intensities at fundamental and SH frequency, proportional to the integrals $I_{F}(q)$ and $I_{S}(q)$ defined by Eq. (49a), are plotted as a function of the transverse wave number (Fig. 6). One immediately notes that fluorescence emission at SH frequency is much weaker than at fundamental frequency. In the region of efficient spontaneous emission, typical values of the ratio $N_{S}(q) / N_{F}(q)$ are of the order of magnitude of 0.1. This low value can be traced back to the fact that twin-photon emission mainly occurs in the region of the crystal where the SH homogeneous wave is strong. But there, the fundamental pump field, which a)

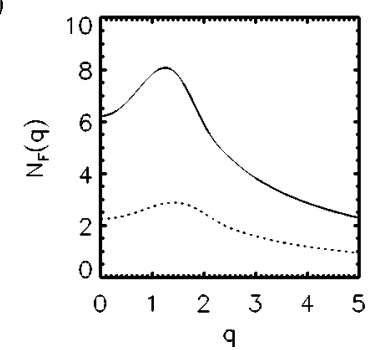

b)

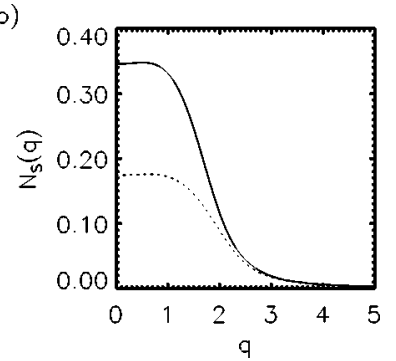

FIG. 6. Mean intensities of spontaneous emission at fundamental (a) and SH frequency (b) as a function of the transverse wave number. Interaction length: $z=1.5$ (dotted line) and $z=2$ (solid line), corresponding to $82 \%(z=1.5)$, resp. $93 \%(z=2)$ SHG efficiency.

acts as a pump also for the up-conversion process, is strongly depleted (Fig. 1) and therefore, the conversion of the fluorescence field from $\omega$ up to $2 \omega$ is not efficient. At first sight, it seems surprising that the profile of the transverse distribution of fluorescence field at fundamental frequency shows a maximum at a finite transverse wave number. One would rather expect, in the perfect phase-matched case, the spontaneous emission to be strongest when both twin photons are emitted collinearly in the pump beam direction, as predicted and demonstrated experimentally for the type I OPA [63]. The effect observed here is a consequence of the variation of the temporal bandwidth of photon down-conversion with the transverse wave number. To see this, we start from the propagation equation for the fundamental field, Eq. (22a), in which we neglect the contribution coming from the copropagating SH field. This approximation is justified by the different magnitudes of spontaneous emission at fundamental and $\mathrm{SH}$ frequency (see Fig. 6). One is left with an equation

$$
\frac{\partial}{\partial z} \hat{a}_{F}(z, \vec{q}, \Omega)=-c_{S}(z) \hat{a}_{F}^{\dagger}(z,-\vec{q},-\Omega) e^{-i \Delta(\vec{q}, \Omega) z},
$$

which describes an OPA with a $z$-dependent pump. (From now on, in order to simplify the notation, we will omit the "tildes" above the dimensionless quantities and the arrows above the wave vectors.) The effective phase mismatch $\Delta(\vec{q}, \Omega)$ being responsible for an oscillatory phase modulation on the rhs of Eq. (51), the production of pairs of fundamental photons with frequencies $(q, \Omega)$ and $(-q,-\Omega)$ should be the most efficient if the condition $\Delta(q, \Omega)=0$ is fulfilled. This clearly appears in the case of the OPA in the undepleted pump approximation [55,63], for which $\vec{V}_{F}^{*}(q, \Omega) \cdot \vec{V}_{F}(q, \Omega)$, which can be identified with the spectral density of spontaneous emission at fundamental frequency, can be calculated analytically:

$$
\left.\vec{V}_{F}^{*}(q, \Omega) \cdot \vec{V}_{F}(q, \Omega)\right|_{O P A}=\left(\frac{\sinh \left(\sqrt{1-\Delta^{2}(q, \Omega) / 4} z\right)}{\sqrt{1-\Delta^{2}(q, \Omega) / 4}}\right)^{2} .
$$

In Ref. [63], the fluorescence spectrum was investigated at degeneracy, i.e., for $\Omega=0$. Experimentally this was achieved by putting a monochromator in front of the detector and, in 

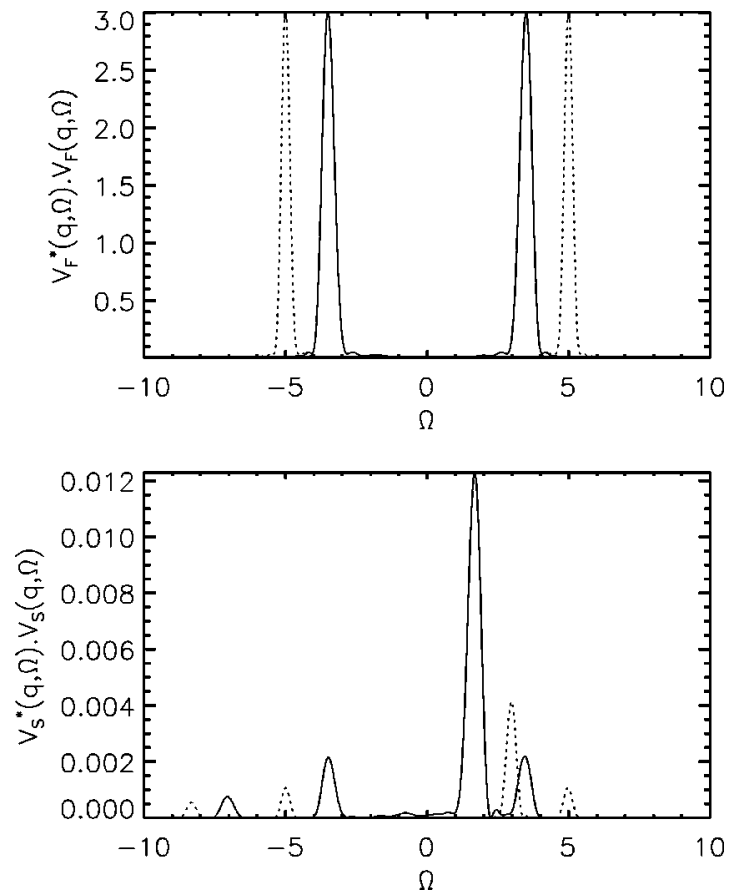

FIG. 7. Spectral density of spontaneous emission at fundamental (upper panel) and SH frequency (lower panel) as a function of the frequency offset $\Omega$, for two different values of the transverse wave number: $q=3.5$ (solid line) and $q=5$ (dotted line). Interaction length: $z=2$.

this case, the effective phase mismatch $\Delta(q, \Omega=0)$ indeed vanishes at $q=0$, giving a maximal spontaneous emission along the beam axis. Here, we consider the whole fluorescence spectrum, i.e., integrating over all temporal frequencies. The upper plot of Fig. 7 represents the spectral density of spontaneous emission at fundamental frequency as a function of the frequency offset $\Omega$. As in the case of the OPA in the undepleted pump approximation, for a given value of $q$, $\vec{V}_{F}^{*}(q, \Omega) \cdot \vec{V}_{F}(q, \Omega)$ is essentially nonzero if $\Omega$ is inside a region formed by two intervals centered on $+q$ and $-q$, which precisely ensure that $\Delta(q, \Omega)=0$. Starting from $q$ $=0$, for which the two intervals perfectly overlap, the width of this region, in terms of $\Omega$, first increases with $q$, until the two intervals around $+q$ and $-q$ start to be separated by a central region of low spectral density. Further increasing $q$ leads again to a reduction of the total width of the region of effective spontaneous emission, since it scales like $1 / q$, as shown by a elementary inspection of Eq. (28a). Considering Eq. (52) reveals that this bandwidth is inversely proportional to the square root of the propagation length $z$. As a consequence, the maximum of the fluorescence spectrum is shifted to smaller wave numbers, when the propagation length increases, as can be seen from Fig. 6 .

No similar behavior is observed in the fluorescence spectrum at SH frequency which instead shows a plateau at small $q$, followed by a monotonous decrease. To understand this behavior, it is again useful to consider the corresponding spectral density $\vec{V}_{S}^{*}(q, \Omega) \cdot \vec{V}_{S}(q, \Omega)$ (lower panel of Fig. 7), which reveals an interesting structure: four peaks can be distinguished. Two of them are located at $\Omega= \pm q$, and coincide with the maxima of the spectral density of emission at fundamental frequency. This is consistent with the expectation that a maximal twin-photon emission should translate into an enhancement of fluorescence at SH frequency. However, two additional peaks are present, one of them being the dominant contribution to spontaneous emission at $2 \omega$. A closer inspection reveals that these peaks are centered around two frequencies

$$
\Omega_{ \pm}^{\max }=\frac{1}{3}\left(-2 \eta \pm \sqrt{4 \eta^{2}+9 q^{2}}\right),
$$

which fulfill the condition:

$$
D\left(q, \Omega_{ \pm}^{\max }\right)=\Delta\left(q, \Omega_{ \pm}^{\max }\right),
$$

i.e., for which the two effective phase mismatches defined by Eqs. (28a) and (28b) coincide. Coming back to the coupled propagation equations (22a) and (22b), condition (54) implies that up-conversion is perfectly phase matched with twin-photon emission at any point of the crystal. Obviously, this gives the condition for an optimal conversion of the spontaneously generated fundamental field up to SH frequency. This conclusion is consistent with recent results of a numerical study of colored conical emission in SHG [47]. As a consequence of the group velocity mismatch between fundamental and SH field, $\Omega_{+}^{\max }$ and $\Omega_{-}^{\max }$ are not symmetrical with respect to the origin. Since $\left|\Delta\left(q, \Omega_{+}^{\max }\right)\right|$ $<\left|\Delta\left(q, \Omega_{-}^{\max }\right)\right|$, the twin-photon emission is less efficient at $\left(q, \Omega_{-}^{\max }\right)$ than at $\left(q, \Omega_{+}^{\max }\right)$ and, as a result, the spectral density $\vec{V}_{S}^{*}(q, \Omega) \cdot \vec{V}_{S}(q, \Omega)$ shows a higher peak at $\Omega_{+}^{\max }$ than at $\Omega_{-}^{\max }$. At small $q$ the peaks at $\Omega_{+}^{\max }$ and at $q$ roughly coincide, resulting in the plateau in Fig. 7. When $q$ increases, the region of maximal SH fluorescence is shifted towards higher frequencies, as predicted by Eq. (53), with a strong decrease of the maximum value of the spectral density. As a consequence, the intensity of the SH fluorescence field rapidly drops to zero at higher transverse wave numbers.

As next, the statistics of the individual currents are briefly investigated. The normalized photon number variances (50a) can be rewritten using identity (35) as

$$
\frac{\left\langle\hat{N}_{i}(q)^{2}\right\rangle-\left\langle\hat{N}_{i}(q)\right\rangle^{2}}{\mathcal{Z}_{S N}}=1+\frac{\int d \Omega\left[\vec{V}_{i}^{*}(q, \Omega) \cdot \vec{V}_{i}(q, \Omega)\right]^{2}}{\int d \Omega\left[\vec{V}_{i}^{*}(q, \Omega) \cdot \vec{V}_{i}(q, \Omega)\right]}
$$

and, since the fraction is positive, the individual photocurrents are always superpoissonian. Taking into account the peak structure of both spectral densities, Eq. (55) can be approximated as

$$
\frac{\left\langle\hat{N}_{i}(q)^{2}\right\rangle-\left\langle\hat{N}_{i}(q)\right\rangle^{2}}{\mathcal{Z}_{S N}}=1+\left.\alpha\left[\vec{V}_{i}^{*}(q, \Omega) \cdot \vec{V}_{i}(q, \Omega)\right]\right|_{\max },
$$

where $\left.\left(\vec{V}_{i}^{*}(q, \Omega) \cdot \vec{V}_{i}(q, \Omega)\right)\right|_{\max }$ is the maximum value of the spectral density with respect to $\Omega$ for a given $q$ and $\alpha$ is 
a)

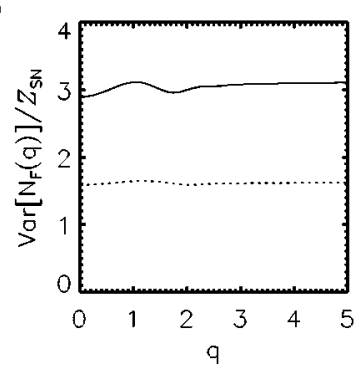

b)

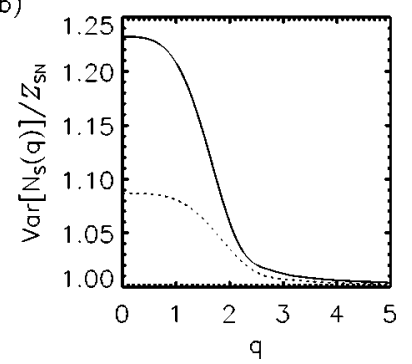

FIG. 8. Level of noise, normalized to the shot noise $\left(Z_{\mathrm{SN}}\right)$, in the individual currents at fundamental (a) and SH frequency (b) as a function of the transverse wave number. Interaction length: $z$ $=1.5$ (dotted line), $z=2$ (solid line).

a numerical factor depending on the specific shape of the peaks. The noise level in the individual current is hence essentially determined by the maximal value of the spectral density, which characterizes the strength of the amplification of the vacuum fluctuations in the mode with frequencies $(\Omega, q)$. These quantities have been plotted in Fig. 8 as a function of the transverse wave number.

At fundamental frequency, the amount of excess noise is almost independent of the transverse wave number, and Eq. (56) shows that this property is due to the fact that changing the value of the wave number does not affect the maximum value of the spectral density (see Fig. 7). Again, this is not true at $\mathrm{SH}$ frequency, for which a higher wave number translates into a lower maximal spectral density of fluorescence and hence a noise level closer to the shot noise.

The interesting feature of the fluorescence field of an OPA, as pointed out in Ref. [48], relies on the fact that although the individual photocurrents collected in two symmetrical parts of the far-field plane can be both well above the shot noise, they show perfectly correlated quantum fluctuations. In the case of TW-SHG, the photon up-conversion mechanism deteriorates the correlations between the fluctuations of $\hat{N}_{F}(q)$ and $\hat{N}_{F}(-q)$, and gives rise to a finite level of quantum noise in the difference $\hat{N}_{F}(q)-\hat{N}_{F}(-q)$. This quantity has been plotted in Fig. 9, together with the upconversion rate for comparison. Whereas in the simplified model, these two quantities were shown to exactly coincide [see Eq. (11)], we observe that in TW-SHG the noise level in the difference $\hat{N}_{F}(q)-\hat{N}_{F}(-q)$ is below the up-conversion rate, i.e., the deterioration of the twin-beam correlations is
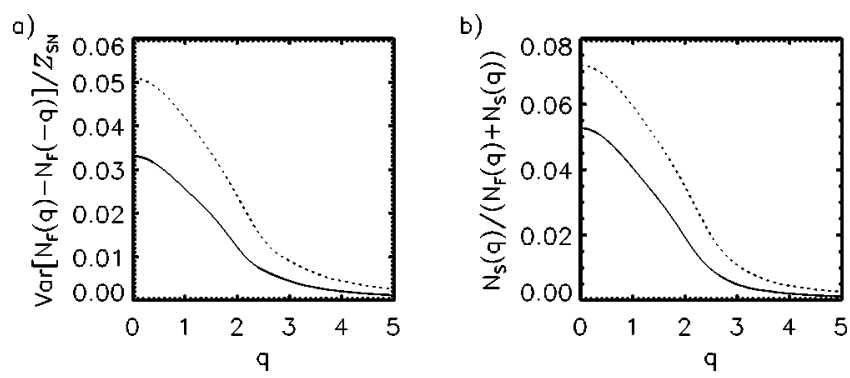

FIG. 9. Twin-beam correlation at fundamental frequency (a) and up-conversion rate (b). Interaction length: $z=1.5$ (dotted line), $z$ $=2($ solid line $)$

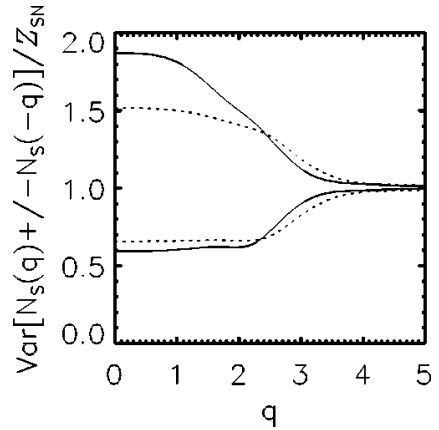

FIG. 10. Noise level, normalized to the shot noise $\left(Z_{\mathrm{SN}}\right)$, in the sum (upper curves) and difference (lower curves) of the $\mathrm{SH}$ far-field intensities at opposite wave vectors, as a function of the transverse wave number. Interaction length: $z=1.5$ (dotted lines) and $z=2$ (solid lines).

less pronounced than predicted by the simplified model. As already pointed out, in TW-SHG, twin photon production and up-conversion take place simultaneously. This mixing implies that TW-SHG, instead of the simplified model represented in Fig. 3, is more similar to a cascade of many of those simplified models. The difference is that what enters into each BS at SH frequency, instead of being the vacuum fluctuations (Fig. 3), is in fact the output of the previous "simplified model" in the cascade, and hence already contains some twin-beam correlations. Therefore it seems reasonable that spatial mixing finally generates "more correlations" than if the two processes were spatially separated. Increasing the transverse wave number, the up-conversion becomes less efficient and therefore, the deterioration of the perfect twin beam correlations at fundamental frequency is less pronounced. A similar effect occurs when the propagation length increases (Fig. 9): in this case, the intensity of spontaneous emission at fundamental frequency increases faster than the one at $\mathrm{SH}$ frequency and the degradation of the twin-beam correlations is again reduced.

As far as the presence of twin-beam correlations at $\mathrm{SH}$ frequency is concerned, the noise level in the difference $\hat{N}_{S}(q)-\hat{N}_{S}(-q)$, plotted in Fig. 10, is clearly below the shot noise: the partial up-conversion of the twin photons generated at fundamental frequency transfers a part of the twinbeam correlations up to SH frequency. As can easily be checked, $\operatorname{Var}\left(\hat{N}_{S}(q)-\hat{N}_{S}(-q)\right) / \mathcal{Z}_{S N}$ is below the value of the ratio $\left\langle\hat{N}_{F}(q)\right\rangle /\left(\left\langle\hat{N}_{F}(q)\right\rangle+\left\langle\hat{N}_{S}(q)\right\rangle\right)$, and therefore the simplified model underestimates the amount of twin-beam correlations present at $\mathrm{SH}$ frequency.

Finally, we looked for spatial correlations between the two fields. The noise levels in the sum and the difference of the two photocurrents at the same far-field point $N_{F}(q)$ $\pm N_{S}(q)$ and at symmetric points $N_{F}(q) \pm N_{S}(-q)$ are shown in Fig. 11. Similarly to what was observed in the cavity case [40], the fluctuations of the intensities $\hat{N}_{F}(q)$ and $\hat{N}_{S}(-q)$ turn out to be more strongly correlated than the intensities at the same far-field point $\hat{N}_{F}(q)$ and $\hat{N}_{S}(q)$. As developed in Sec. III, this observation finds a simple explanation in terms of partial noise cancellation from the recombination of those twin-photon pairs, which were broken 

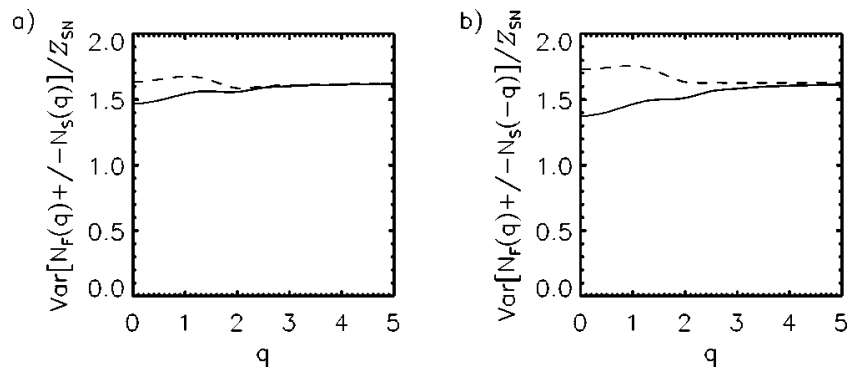

FIG. 11. Cross correlations (difference: solid curve; sum: dashed curve) between the intensity fluctuations at fundamental and $\mathrm{SH}$ frequency, at the same transverse wave vector (a) and at opposite wave vectors (b), as a function of the transverse wave number. Interaction length: $z=1.5$.

through an up-conversion process.

However, in contrast to the cavity case [40], neither $\hat{N}_{F}(q)-\hat{N}_{S}(-q)$ nor $\hat{N}_{F}(q)-\hat{N}_{S}(q)$ is below the shot noise. In fact, a comparison of Fig. 11 with the dotted curves of Fig. 8 reveals that these quantities are manifestly dominated by the noise level in the individual photocurrent at fundamental frequency. This obviously originates from the fact that $\left\langle\hat{N}_{S}( \pm q)\right\rangle \ll\left\langle\hat{N}_{F}(q)\right\rangle$, as pointed out at the beginning of this section, so that $\operatorname{Var}\left(N_{F}(q)-N_{S}( \pm q)\right) / \mathcal{Z}_{S N}$ $\simeq \operatorname{Var}\left(N_{F}(q)\right) / \mathcal{Z}_{S N}$. In this particular situation, the strongest correlations are found not between $\hat{N}_{F}(+q)$ and $\hat{N}_{S}( \pm q)$ but between $\hat{N}_{F}(+q)$ and a scaled version of $\hat{N}_{S}( \pm q)$. Following the ideas introduced in Ref. [64] and exploited in Ref. [22] for the investigation of Einstein-Podolsky-Rosen Correlations in the OPO, we investigated the statistics of the linear combination of photocurrents $\hat{N}_{F}(+q)+\lambda \hat{N}_{S}( \pm q)$. Plotting the corresponding noise levels, as always normalized to the shot noise, as a function of the scaling parameter $\lambda$ (Fig. 12), we find that for a proper choice of the parameter $\lambda$, the quantity $\hat{N}_{F}(+q)+\lambda \hat{N}_{S}(-q)$ indeed drops below the shot noise, revealing the presence of correlations of nonclassical nature between the two fields at opposite wave numbers, whereas $\hat{N}_{F}(+q)+\lambda \hat{N}_{S}(+q)$ is at best slightly above the shot noise. Figure 13 shows the value of the noise level in $\hat{N}_{F}(q)+\lambda \hat{N}_{S}( \pm q)$, minimized for each wave number with respect to $\lambda[65]$.

It can be interesting to carry out the same optimization procedure in the case of the simplified model considered in Sec. III, and we found the following analytical results: a)

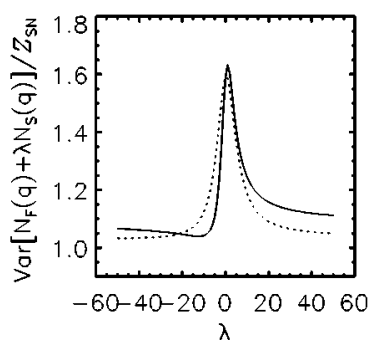

b)

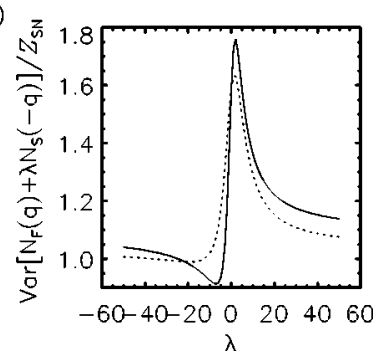

FIG. 12. Weighted cross correlation as a function of the weighting parameter $\lambda$. Interaction length: $z=1.5$. Transverse wave numbers: $q=0$ (solid line) and $q=2$ (dotted line).
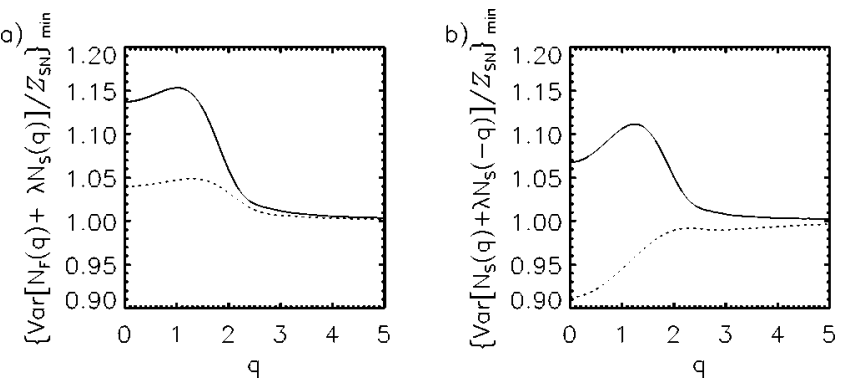

FIG. 13. Optimized cross correlation as a function of the transverse wave number, at the same transverse wave vector (a) and opposite wave vectors (b). Propagation length $z=1.5$ (dotted line) and $z=2$ (solid line).

$$
\begin{gathered}
\left.\frac{\operatorname{Var}\left(\hat{N}_{F,+\vec{q}}+\lambda \hat{N}_{S,+\vec{q}}\right)}{\mathcal{Z}_{S N}}\right|_{\min (\lambda)}=1, \\
\left.\frac{\operatorname{Var}\left(\hat{N}_{F,+\vec{q}^{+}}+\lambda \hat{N}_{S,-\vec{q})}\right.}{\mathcal{Z}_{S N}}\right|_{\min (\lambda)}=1-2 t^{2} r^{2},
\end{gathered}
$$

the last expression being derived under the simplifying assumption that $v \gg 1$. The noise level in a linear combination of both outputs at a given wave vector is at best given by the shot-noise level, whereas the fluctuations in a linear combination of $\hat{N}_{F,+\vec{q}}$ and $\hat{N}_{S,-\vec{q}}$ can drop below this limit, the difference $-2 t^{2} r^{2}$ being precisely the amount of noise cancellation coming from twin-photon pair recombination. In both cases, the value of $\lambda_{\min }$ which minimizes the noise level is given by $-t^{2} / r^{2}$ and coincides with the intensity ratio $\left\langle\hat{N}_{F, q}\right\rangle /\left\langle\hat{N}_{S, q}\right\rangle$. This simple result corroborates the interpretation of $\lambda_{\min }$ as a factor which compensates the difference between the average values of the involved currents, whereas the negative sign of $\lambda_{\text {min }}$ means that the currents have to be substracted from each other. Coming back to the full multimode calculation, the optimal value $\lambda_{\text {min }}$ can be easily calculated in terms of the different integrals defined by Eqs. (49), and has been plotted in Fig. 14. A slight deviation from the ratio $\left\langle\hat{N}_{F}(q)\right\rangle /\left\langle\hat{N}_{S}(q)\right\rangle$ predicted for the simplified model is observed. A further discrepancy between the two models is seen in the fact that while the simplified model predicts the optimized variance of $\hat{N}_{F}(+q)+\lambda_{\min } \hat{N}_{S}(-q)$ o)

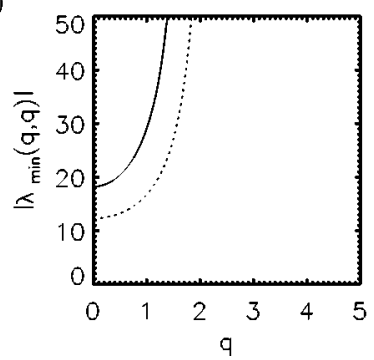

b)

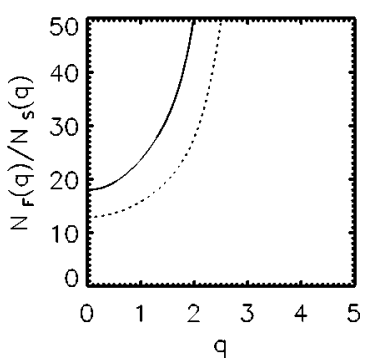

FIG. 14. Value of the parameter $\lambda$, which minimizes the cross correlation at the same wave vector (a) and ratio of the intensities at fundamental and SH frequency (b). Interaction length: $z=1.5$ (dotted line) and $z=2$ (solid line). 
to be below the shot-noise level [Eq. (57b)], the full calculation shows that above a certain propagation length, this variance remains above the shot noise. This is connected to the fact that $\hat{N}_{F}(q)+\lambda_{\min } \hat{N}_{S}(q)$ displays some excess noise at small transverse wave numbers, missed in the simplified model, which increases with the propagation length. As a result, for larger propagation lengths, the partial noise cancellation present in $\hat{N}_{F}(q)+\lambda_{\min } \hat{N}_{S}(-q)$ is not able to compensate this excess noise and this quantity remains above shot noise. However, the optimization procedure presented here allows a substantial reduction of the noise level in the difference of intensity of the two fields with respect to the equally weighted difference of photocurrents.

\section{CONCLUSION}

In this paper, we investigated the properties of the fluorescence field generated by phase matched second-harmonic generation in the traveling-wave configuration. It was first shown that spontaneous emission leads to the generation of fluorescence field at fundamental and SH frequency, as the result of two different mechanisms: first the strong SH wave generated from the pump field through SHG decays into pairs of twin photons. Subsequently, this fluorescence field may undergo a partial up-conversion triggered by the strongly depleted pump field at fundamental frequency, and gives rise to the fluorescence field at SH frequency. However, in the case of phase-matched SHG, the depletion of the pump implies that this field distribution always remains much weaker than the fluorescence field at fundamental frequency. We studied the transverse far-field intensity distribution at each frequency and explained the results in terms of the spectral density of spontaneous emission at each frequency. We then focused on the study of the spatial quantum correlations in the fluctuations of the far-field intensities. We found that the intensity of the fluorescence field at fundamental frequency collected in symmetrical portions of the far-field plane displays very strongly correlated fluctuations. Unlike the OPA, the noise level in the difference is not rigorously vanishing, but is found to be approximately given by the up-conversion rate at the wave number considered. Twinbeam correlations were also predicted at SH frequency. The originality of these correlations is that they cannot be traced back to a twin-photon emission process. The emergence of these nonclassical correlations originates from the fact that the considered beams are the result of a secondary process acting on twin beams, and hence display some correlations present in the field from which they are generated. Other interesting correlations were predicted between the fluctuations of the intensities of fundamental and $\mathrm{SH}$ field either at the same transverse wave vector, or at opposite wave vectors. These cross correlations, interestingly, were found to be stronger when the intensities are compared at opposite farfield points than at the same point. We showed that these counterintuitive features could be understood in terms of partial noise cancellation due to the recombination of twinphoton pairs broken by the secondary process. This was illustrated from a qualitative point of view by considering a simplified few-mode model based on the formal equivalence between up-conversion and beam splitters. This approach could be useful to understand the quantum correlations in other optical systems, in which twin-photon emission competes with secondary processes. In particular, these considerations could be also adapted to the cavity configuration.

\section{ACKNOWLEDGMENTS}

Financial support from the European Commission Project QUANTIM (Grant No. IST-2000-26019), as well as from CONOCE: MCyT (Spain) (Project No. BFM2000-1108) is acknowledged. Stimulating discussions with Maxi San Miguel as well as his interest in this work are acknowledged. The author is also grateful to Morten Bache for his careful reading of the manuscript and many constructive remarks.
[1] D.N. Klyshko, Photons and Nonlinear Optics (Gordon and Breach, New York, 1988).

[2] L. Mandel and E. Wolf, Optical Coherence and Quantum Optics (Cambridge, New York, 1995).

[3] A. Yariv, Quantum Electronics, 3rd ed. (Wiley, New York, 1988).

[4] D. Burnham and D. Weinberg, Phys. Rev. Lett. 25, 84 (1970).

[5] S. Friberg, C. Hong, and L. Mandel, Phys. Rev. Lett. 54, 2011 (1985).

[6] B. Mollow, Phys. Rev. A 49, 2684 (1973).

[7] A. Einstein, B. Podolsky, and N. Rosen, Phys. Rev. 47, 777 (1935).

[8] J.S. Bell, Physics (Long Island City, N.Y.) 1, 195 (1964).

[9] A. Zeilinger, Rev. Mod. Phys. 71, 288 (1999).

[10] A.F. Abouraddy, B.E.A. Saleh, A.V. Sergienko, and M.C. Teich, Phys. Rev. Lett. 87, 123602 (2001).

[11] A.N. Boto, P. Kok, D.S. Abrams, S.L. Braunstein, C.P. Williams, and J.P. Dowling, Phys. Rev. Lett. 85, 2733 (2000).
[12] A.K. Ekert, Phys. Rev. Lett. 67, 661 (1991).

[13] A.V. Sergienko, M. Atature, Z. Walton, G. Jaeger, B.E.A. Saleh, and M.C. Teich, Phys. Rev. A 60, R2622 (1999).

[14] C.H. Bennett, G. Brassard, C. Crepeau, R. Jozsa, A. Peres, and W.K. Wootters, Phys. Rev. Lett. 70, 1895 (1993).

[15] D. Bouwmeester, J.W. Pan, K. Mattle, M. Eibl, H. Weinfurter, and A. Zeilinger, Nature (London) 390, 575 (1997).

[16] A. Zeilinger, Phys. World 11, 35 (1998).

[17] S. Reynaud, C. Fabre, and E. Giacobino, J. Opt. Soc. Am. B 4, 1520 (1987).

[18] S. Reynaud, Europhys. Lett. 4, 427 (1987).

[19] E. Giacobino, C. Fabre, A. Heidmann, R. Horowicz, and S. Reynaud, in Fundamentals of Quantum Optics II, edited by F. Ehlotzky (Springer-Verlag, New York, 1987).

[20] A. Heidmann, R.J. Horowicz, S. Reynaud, E. Giacobino C. Fabre, and G. Camy, Phys. Rev. Lett. 59, 2555 (1987).

[21] G.L. Oppo, M. Brambilla, and L.A. Lugiato, Phys. Rev. A 49, 2028 (1994). 
[22] F. Castelli and L.A. Lugiato, J. Mod. Opt. 44, 765 (1997).

[23] J.B. Geddes, J.V. Moloney, E.M. Wright, and W.J. Firth, Opt. Commun. 111, 69 (1993).

[24] M. Hoyuelos, P. Colet, M. San Miguel, and D. Walgraef, Phys. Rev. E 58, 2992 (1998).

[25] M. Hoyuelos, A. Sinatra, P. Colet, L.A. Lugiato, and M. San Miguel, Phys. Rev. A 59, 1622 (1999).

[26] R. Zambrini, M. Hoyuelos, A. Gatti, P. Colet, L. Lugiato, and M. San Miguel, Phys. Rev. A 62, 063801 (2000).

[27] R. Graham, Phys. Rev. Lett. 52, 117 (1984).

[28] R. Zambrini and M. San Miguel, Phys. Rev. A 66, 023807 (2002).

[29] H. Ward, M.N. Ouarzazi, M. Taki, and P. Glorieux, Phys. Rev. E 63, 016604 (2000).

[30] R. Zambrini, S. Barnett, P. Colet, and M. San Miguel, Eur. Phys. J. D (to be published).

[31] M. Santagiustina, P. Colet, M. San Miguel, and D. Walgraef, Phys. Rev. Lett. 79, 3633 (1997).

[32] M. Santagiustina, P. Colet, M. San Miguel, and D. Walgraef, Opt. Lett. 23, 1167 (1998).

[33] R. Zambrini, S.M. Barnett, P. Colet, and M. San Miguel, Phys. Rev. A 65, 023813 (2002).

[34] C. Schwob, P.F. Cohadon, C. Fabre, M.A.M. Marte, H. Ritsch, A. Gatti, and L. Lugiato, Appl. Phys. B: Lasers Opt. 66, 685 (1998)

[35] W.J. Firth, A.J. Scroggie, G.S. MacDonald, and L.A. Lugiato, Phys. Rev. A 46, R3609 (1992).

[36] G. Grynberg and L.A. Lugiato, Opt. Commun. 101, 69 (1993).

[37] A. Gatti and S. Mancini, Phys. Rev. A 65, 013816 (2001).

[38] D. Gomila and P. Colet, Phys. Rev. E 66, 046223 (2002).

[39] M. Hoyuelos, G.L. Oppo, P. Colet, and M. San Miguel, Eur. Phys. J. D (to be published).

[40] M. Bache, P. Scotto, R. Zambrini, M. San Miguel, and M. Saffman, Phys. Rev. A 66, 013809 (2002).

[41] P.L. Ramazza, S. Ducci, A. Zavatta, M. Bellini, and F.T. Arrechi, Appl. Phys. B: Lasers Opt. B75, 53 (2002), and references quoted therein.

[42] P. Scotto and M. San Miguel, Phys. Rev. A 65, 043811 (2002).

[43] J.A. Armstrong, N. Bloembergen, J. Ducuing, and P.S. Pershan, Phys. Rev. 127, 1918 (1962).

[44] M.K. Olsen, R.J. Horowicz, L.I. Plimak, N. Treps, and C. Fabre, Phys. Rev. A 61, 021803 (2000).

[45] Equation (1) takes into account any pair of photons with frequencies $\omega_{1}=\omega+\Omega$ and $\omega_{2}=\omega-\Omega$ fulfilling energy conservation $\omega_{1}+\omega_{2}=2 \omega$. The phase-matching condition will imply, however, that this process will only be efficient in a time frequency range $\Omega \ll \omega$.

[46] S. Trillo and P. Ferro, Opt. Lett. 20, 438 (1995).

[47] S. Trillo, C. Conti, P. Di Trapani, O. Jedrkiewicz, J. Trull, G. Valiulis, and G. Bellanca, Opt. Lett. 27, 1451 (2002).
[48] E. Brambilla, A. Gatti, L.A. Lugiato, and M.I. Kolobov, Eur. Phys. J. D 15, 127 (2001).

[49] A. Andreoni, M. Bondani, G.M. D’Ariano, and M.G.A. Paris, Eur. Phys. J. D 13, 415 (2001).

[50] G.M. D'Ariano, M.G.A. Paris, and M.F. Sacchi, Nuovo Cimento Soc. Ital. Fis., B 114B, 339 (1999).

[51] C. Fabre, in Proceedings of the Les Houches Summer School, Session 63 (Elsevier, Amsterdam, 1997), p. 181.

[52] M.J. Collett and D.F. Walls, Phys. Rev. A 32, 2887 (1985).

[53] M. Bache, Yu.B. Gaididei, and P.L. Christiansen, Phys. Rev. A 67, 043802 (2003).

[54] S. Reynaud, A. Heidmann, E. Giacobino, and C. Fabre, Progress in Optics XXX (Elsevier Science, Amsterdam, 1992), p. 1.

[55] M.I. Kolobov, Rev. Mod. Phys. 71, 1539 (1999).

[56] T.A.B. Kennedy and S. Trillo, Phys. Rev. A 54, 4396 (1996).

[57] Z.Y. Ou, Phys. Rev. A 49, 2106 (1994).

[58] In principle, other three-wave mixing processes may also occur inside the NL crystal, such as the sum-frequency mixing of two off-axis fundamental photons $[\omega](q, \Omega)+[\omega]\left(q^{\prime}, \Omega^{\prime}\right)$ $\rightarrow[2 \omega]\left(q+q^{\prime}, \Omega+\Omega^{\prime}\right)$. However, assuming that the spontaneously emitted fields are at any point of the crystal much weaker than the coherent homogeneous waves at fundamental and $\mathrm{SH}$ frequency, those processes which do not involve at least one on-axis photon $[\omega](00)$ or $[2 \omega](00)$ can be neglected. Mathematically, this assumption is encoded in the linearization of the propagation equations for the quantum fields.

[59] A description of field dynamics based on a linearization around homogeneous modes predicts correlations between transverse modes associated either with the same or with opposite wave vectors. Correlations between modes with wave vectors different in modulus would only appear if nonlinear corrections to the linearized approach would become important. This was already pointed out in Ref. [40] for the cavity case.

[60] In the Heisenberg picture, the evolution of the system of the coupled quantum fields during propagation is encoded in the dependence of the field operators on the propagation length, while the quantum state of the system is simply given by the initial state of the system, which is here the vacuum state. While normally ordered expectation values of any combination of input operators vanish, the same quantity for output operators in general does not.

[61] M.I. Kolobov and L.A. Lugiato, Phys. Rev. A 52, 4930 (1995).

[62] M.K. Olsen and R.J. Horowicz, Opt. Commun. 168, 135 (1999).

[63] F. Devaux and E. Lantz, Eur. Phys. J. D 8, 117 (2000).

[64] M.D. Reid, Phys. Rev. A 40, 913 (1989).

[65] Applying the same procedure to the twin beam correlations, i.e., considering the quantities $N_{i}(+q)+\lambda_{i} N_{i}(-q)$, we checked that the noise level is always minimal when the intensities are equally weighted (i.e., for $\lambda=-1$ ). 